Flow, Turbulence and Combustion manuscript No.

(will be inserted by the editor)

\title{
Towards the development of an evolution equation for flame turbulence interaction in premixed turbulent combustion
}

\author{
U. Ahmed · R. Prosser · A. J. Revell
}

Received: date / Accepted: date

\begin{abstract}
Flame turbulence interaction is one of the leading order terms in the scalar dissipation $\left(\widetilde{\varepsilon}_{c}\right)$ transport equation [35] and is thus an important phenomenon in premixed turbulent combustion. Swaminathan and Grout [36] and Chakraborty and Swaminathan [15, 16] have shown that the effect of strain rate on the transport of $\widetilde{\varepsilon}_{c}$ is dominated by the interaction between the fluctuating scalar gradients and the fluctuating strain rate, denoted here by $\bar{\rho} \widetilde{\Delta}_{c}=\overline{\rho \alpha \nabla c^{\prime \prime} S_{i j}^{\prime \prime} \nabla c^{\prime \prime}}$; this represents the flame turbulence interaction. In order to obtain an accurate representation of this phenomenon, a new evolution equation for $\widetilde{\Delta}_{c}$ has been proposed. This equation gives a detailed insight into flame turbulence interaction and

U. Ahmed

Modelling \& Simulation Centre, School of MACE, University of Manchester, M13 9PL, UK

E-mail: umair.ahmed@manchester.ac.uk

R. Prosser

Modelling \& Simulation Centre, School of MACE, University of Manchester, M13 9PL, UK

E-mail: robert.prosser@manchester.ac.uk

A. J. Revell

Modelling \& Simulation Centre, School of MACE, University of Manchester, M13 9PL, UK

E-mail: alistair.revell@manchester.ac.uk
\end{abstract}


provides an alternative approach to model the important physics represented by $\widetilde{\Delta}_{c}$. The $\widetilde{\Delta}_{c}$ evolution equation is derived in detail and an order of magnitude analysis is carried out to determine the leading order terms in the $\widetilde{\Delta}_{c}$ evolution equation. The leading order terms are then studied using a Direct Numerical Simulation (DNS) of premixed turbulent flames in the corrugated flamelet regime. It is found that the behaviour of $\widetilde{\Delta}_{c}$ is determined by the competition between the source terms (pressure gradient and the reaction rate), diffusion/dissipation processes, turbulent strain rate and the dilatation rate. Closures for the leading order terms in $\widetilde{\Delta}_{c}$ evolution equation have been proposed and compared with the DNS data.

Keywords Flame turbulence interaction $\cdot$ Scalar turbulence interaction $\cdot$ Premixed turbulent combustion $\cdot$ Scalar dissipation

\section{Introduction}

In premixed turbulent combustion modelling, the closure for the reaction rate is challenging. The problem can be simplified under the thin flamelet assumption, according to which the reaction zone is assumed be thin compared to the Kolmogorov length scale [7]. In the flamelet approach it is assumed that the flame consists of a very thin sheet, and its local structure is the same as that of a laminar flame [8,9]. A laminar flamelet consists of a thin and highly wrinkled interface separating products from reactants. This interface contains all the effects of the combustion chemistry associated with heat release and all of the related molecular transport effects, thus making it possible to decouple the chemistry from the turbulence. The thin flamelet assumption makes it possible to approximate the probability density function (pdf) of a reaction progress variable $(c)$ (usually defined as a normalised product mass fraction $Y$ ), through a double delta function, representing the unburnt and burnt mixtures [8]. This approximation leads to simple closures for the turbulent transport and the 
reaction rate. Usually in engineering applications, a transport equation for the Favre averaged progress variable $\widetilde{c}$ is solved. The transport equation for $\widetilde{c}$ requires closures for mean reaction rate $\left(\overline{\dot{\omega}}_{c}\right)$ and the turbulent transport $\left(\widetilde{u^{\prime \prime} c^{\prime \prime}}\right)$ of $\widetilde{c}$. Several approaches to close the reaction rate are available in the literature, although two approaches are widely used under the thin flamelet assumption; the flame surface density $(\Sigma)$ approach and the scalar dissipation $\left(\widetilde{\varepsilon}_{c}\right)$ approach. Borghi has shown that $\Sigma$ and $\widetilde{\varepsilon}_{c}$ are algebraically related to each other [4], thus representing the same physical phenomenon. In the $\Sigma$ approach $\overline{\dot{\omega}}_{c}$ is closed as [12]:

$$
\bar{\omega}_{c} \simeq \Sigma \rho_{u} u_{L}^{0} I_{0}
$$

where $\rho_{u}$ is the density of the reactants, $u_{L}^{0}$ is the unstrained laminar flame speed, and $I_{0}$ represents a correction factor to account for strain rate and curvature effects on the local laminar flame speed [12]. In the $\widetilde{\varepsilon}_{c}$ approach $\overline{\dot{\omega}}_{c}$ is usually closed as [6]:

$$
\bar{\omega}_{c} \simeq \frac{2}{2 C_{m}-1} \bar{\rho} \widetilde{\varepsilon}_{c}
$$

where $\widetilde{\varepsilon}_{c}$ is the scalar dissipation, $\bar{\rho} \widetilde{\varepsilon}_{c}=\overline{\rho \alpha\left(\nabla c^{\prime \prime} \nabla c^{\prime \prime}\right)}$ (double primes denote the Favre fluctuations), $\alpha$ represents the diffusivity of the progress variable and $C_{m}$ is a model constant. Both $\Sigma$ and $\widetilde{\varepsilon}_{c}$ transport equations have been developed in previous studies and details are available in $[35,12,5,13,30,10]$. Here our aim is to develop an evolution equation for flame turbulence interaction, hence the scalar dissipation approach is only used from here onwards.

A transport equation for $\widetilde{\mathcal{\varepsilon}}_{c}$ has been proposed by Swaminathan and Bray [35]:

$$
\bar{\rho} \frac{D \widetilde{\varepsilon}_{c}}{D t}=D_{1}-D_{2}+T_{1}+T_{2}+T_{3}+T_{4}
$$

where the nomenclature of the right-hand side of Eq (3) reflects the most common usage $[35,15,25]$. Each of the terms in Eq. (3) represent a particular physical process; for example 


$$
T_{3}=\underbrace{-2 \overline{\rho \alpha} \frac{\partial \widetilde{c}}{\partial x_{i}}\left(\widehat{\frac{\partial c^{\prime \prime}}{\partial x_{j}} \frac{\partial u_{i}^{\prime \prime}}{\partial x_{j}}}\right)}_{T_{31}} \underbrace{-2 \overline{\rho \alpha}\left(\frac{\partial c^{\prime \prime} S_{i j}^{\prime \prime} \frac{\partial c^{\prime \prime}}{\partial x_{j}}}{\partial x_{i}}\right)}_{T_{32}} \underbrace{-2 \overline{\rho \alpha}\left(\widehat{\frac{\partial c^{\prime \prime}}{\partial x_{i}} \frac{\partial c^{\prime \prime}}{\partial x_{j}}}\right) \widetilde{S_{i j}}}_{T_{33}}
$$

represents the combined effects of flame turbulence interaction. In Eq. (4) $S_{i j}^{\prime \prime}$ represents the fluctuating strain rate and is defined as $S_{i j}^{\prime \prime}=0.5\left(\partial u_{i}^{\prime \prime} / \partial x_{j}+\partial u_{j}^{\prime \prime} / \partial x_{i}\right)$.

An order of magnitude analysis (OMA) under joint assumptions of high turbulent Reynolds $\left(\operatorname{Re}_{l_{t}}\right)$ and Damköhler $(\mathrm{Da})$ numbers shows term $T_{32}$ to be of leading order [35]; the behaviour of $T_{3}$ is thus dominated by the behaviour of $T_{32}$ [36].

The flame turbulence interaction phenomenon presents a major difficulty in obtaining an accurate closure for both the scalar dissipation $\left(\widetilde{\varepsilon}_{c}\right)$ and the flame surface density $(\Sigma)$, and thus the mean reaction rate $\left(\bar{\omega}_{c}\right)$. Here we present an alternative way for the prediction of flame turbulence interaction in premixed flames.

The paper is organised as follows: in the next section we derive the transport equation for flame turbulence interaction. The order of magnitude analysis is carried out in section 3. The details of the DNS data set are discussed in section 4 along with the data processing method followed in this study. The results are discussed in section 5 and the conclusions are summarised in the last section.

\section{Flame turbulence interaction evolution equation}

In the case of statistically multidimensional flames $T_{32}$ can be decomposed using an eigendecomposition and written as $[25,27]$ :

$$
T_{32}=-\overline{2 \rho \alpha\left|\nabla c^{\prime \prime}\right|^{2}\left(e_{\alpha} \cos ^{2} \theta_{\alpha}+e_{\beta} \cos ^{2} \theta_{\beta}+e_{\gamma} \cos ^{2} \theta_{\gamma}\right)}
$$

where $e_{\alpha}, e_{\beta}$ and $e_{\gamma}$ are the eigenvalues of the turbulent strain rate tensor $S_{i j}^{\prime \prime}$. The eigenvalues are ranked as $e_{\alpha}>e_{\beta}>e_{\gamma}$, with $e_{\alpha}$ being the most extensive principal strain rate and $e_{\gamma}$ 
being the most compressive principal strain rate. The angle between the scalar gradient and the eigenvector associated with $e_{\alpha}$ is denoted by $\theta_{\alpha}$. The source or sink behaviour of $T_{32}$ is thus dependent on the statistics of the alignment between the scalar gradient and the directions of the principal strain rates $[13,25]$. It is well known that in case of the cold turbulence, the scalar gradient preferentially aligns with the most compressive principal strain rate $[2$, 3], thus giving a source contribution from $T_{32}$. In case of flows with intense heat release, it has been shown by Chakraborty and Swaminathan [15], Swaminathan and Grout [36], Kim and Pitsch [24] and Hartung et al [22] that the scalar gradient preferentially aligns with the most extensive principal strain rate. The variation between the alignment characteristics is due to the competition between dilatation rate and turbulent strain rate [36]. In case of strong heat release, the dilatation mostly occurring in the local normal direction overcomes the turbulence effects thus causing the scalar gradient to align with the most extensive strain rate $e_{\alpha}[13,24] ; T_{32}$ becomes negative and dissipates the scalar gradient $[13,22,24]$.

In order to capture the physical phenomenon mentioned above, an evolution equation for flame turbulence interaction can be used. In this section we derive the evolution equation in detail and do an order of magnitude analysis for the evolution equation. The evolution equation can be found by taking the total derivative of :

$$
\rho \frac{D}{D t}\left(\alpha \frac{\partial c}{\partial x_{i}} S_{i j} \frac{\partial c}{\partial x_{j}}\right)
$$

where $\alpha$ is assumed to be constant to reduce the complexity of the problem. Applying the Reynolds decomposition to Eq. (6) and then Favre averaging leads to: 


$$
\begin{aligned}
& \overline{\rho \frac{D}{D t}\left(\alpha \frac{\partial c}{\partial x_{i}} S_{i j} \frac{\partial c}{\partial x_{j}}\right)}=\underbrace{\overline{\rho \alpha \frac{D}{D t}\left(\frac{\partial c^{\prime \prime}}{\partial x_{i}} S_{i j}^{\prime \prime} \frac{\partial c^{\prime \prime}}{\partial x_{j}}\right)}}_{\text {Term } 1}+\underbrace{\overline{\rho \alpha \frac{D}{D t}\left(\frac{\partial c^{\prime \prime}}{\partial x_{i}} \widetilde{S_{i j}} \frac{\partial c^{\prime \prime}}{\partial x_{j}}\right)}}_{\text {Term } 2} \\
& +\overline{\rho \alpha \frac{D}{D t}\left(\frac{\partial \widetilde{c}}{\partial x_{i}} S_{i j}^{\prime \prime} \frac{\partial c^{\prime \prime}}{\partial x_{j}}\right)}+\overline{\rho \alpha \frac{D}{D t}\left(\frac{\partial c^{\prime \prime}}{\partial x_{i}} S_{i j}^{\prime \prime} \frac{\partial \widetilde{c}}{\partial x_{j}}\right)} \\
& \text { Term } 3 \quad \text { Term } 4 \\
& +\underbrace{\rho \alpha \frac{D}{D t}\left(\frac{\partial \widetilde{c}}{\partial x_{i}} \widetilde{S_{i j}} \frac{\partial \widetilde{c}}{\partial x_{j}}\right)} \\
& \text { Term } 5
\end{aligned}
$$

An order of magnitude analysis under the joint assumptions of high $\operatorname{Re}_{l_{t}}$ and $D a$ (similar to that of Swaminathan and Bray [35]) is used to find the leading order terms in Eq. (7).

The order of magnitude analysis is carried out based on the following scaling arguments. The spatial derivatives are scaled by the integral length scale $l_{t}$. The ratio of the integral length scale $l_{t}$ and the root mean square velocity $u^{\prime}$ is used to scale the time derivative. The density is scaled by the density of the reactants $\rho_{u}$. The mean velocities are scaled by a reference velocity $u_{\text {ref }}$. The thermal diffusivity of the mixture is scaled by the laminar flame scales $u_{L}^{0} \delta_{L}^{0}$, where $u_{L}^{0}$ is the unstretched laminar flame speed and $\delta_{L}^{0}$ is the laminar flame thermal thickness. The quantities involving or multiplied by the gradient of progress variable are also scaled with the laminar flame quantities.

According to the scaling arguments the terms in Eq. (7) scale as:

Term 1:

$$
\overline{\rho \alpha} \frac{\partial c^{\prime \prime}}{\partial x_{i}} S_{i j}^{\prime \prime} \frac{\partial c^{\prime \prime}}{\partial x_{j}} \simeq \mathscr{O}\left(\rho_{u}\left(\frac{u_{L}^{0}}{\delta_{L}^{0}}\right)^{2} ; 1\right)
$$

Term 2 :

$$
\overline{\rho \alpha} \frac{\partial c^{\prime \prime}}{\partial x_{i}} \frac{\partial c^{\prime \prime}}{\partial x_{j}} \widetilde{S_{i j}} \simeq \mathscr{O}\left(\rho_{u}\left(\frac{u_{L}^{0}}{\delta_{L}^{0}}\right)^{2} ;\left(\frac{u^{\prime} D a}{u_{r e f}}\right)^{-1}\right)
$$

Term 3:

$$
\overline{\rho \alpha} \widetilde{\frac{\partial c^{\prime \prime}}{\partial x_{j}} S_{i j}^{\prime \prime}} \frac{\partial \widetilde{c}}{\partial x_{i}} \simeq \mathscr{O}\left(\rho_{u}\left(\frac{u_{L}^{0}}{\delta_{L}^{0}}\right)^{2} ;\left(R e_{l_{t}} D a\right)^{-1 / 2}\right),
$$


Term 4 :

$$
\overline{\rho \alpha} \frac{\partial c^{\prime \prime}}{\partial x_{i}} S_{i j}^{\prime \prime} \frac{\partial \widetilde{c}}{\partial x_{j}} \simeq \mathscr{O}\left(\rho_{u}\left(\frac{u_{L}^{0}}{\delta_{L}^{0}}\right)^{2} ;\left(R e_{l_{t}} D a\right)^{-1 / 2}\right)
$$

Term 5 :

$$
\overline{\rho \alpha}\left(\frac{\partial \widetilde{c}}{\partial x_{i}} \widetilde{S_{i j}} \frac{\partial \widetilde{c}}{\partial x_{j}}\right) \simeq \mathscr{O}\left(\rho_{u}\left(\frac{u_{L}^{0}}{\delta_{L}^{0}}\right)^{2} ;\left(\frac{R e_{l_{t}} D a^{2} u^{\prime}}{u_{r e f}}\right)^{-1}\right)
$$

The above order of magnitude analysis suggests that in the thin flamelet limit, $\bar{\rho}(\overbrace{\alpha c_{, i}^{\prime \prime} S_{i j}^{\prime \prime} c_{, j}^{\prime \prime}})$ is a dominant term, and, thus an evolution equation for $\bar{\rho}(\overbrace{\alpha c_{, i}^{\prime \prime} S_{i j}^{\prime \prime} c_{, j}^{\prime \prime}})$ will provide a useful first approximation. Introducing the definition:

$$
\bar{\rho} \widetilde{\Delta}_{c} \equiv \overline{\rho \alpha \frac{\partial c^{\prime \prime}}{\partial x_{i}} S_{i j}^{\prime \prime} \frac{\partial c^{\prime \prime}}{\partial x_{j}}}
$$

The total derivative of the fluctuating part of flame turbulence interaction term is :

$$
\begin{aligned}
\overline{\rho \frac{D}{D t}\left(\alpha \frac{\partial c^{\prime \prime}}{\partial x_{i}} S_{i j}^{\prime \prime} \frac{\partial c^{\prime \prime}}{\partial x_{j}}\right)}=\overline{\rho \alpha} \frac{\partial c^{\prime \prime} S_{S_{i j}} \frac{D}{D t}\left(\frac{\partial c^{\prime \prime}}{\partial x_{j}}\right)}{+\rho \alpha}+\overline{\frac{\partial c^{\prime \prime}}{\partial x_{j}} \frac{\partial c^{\prime \prime}}{\partial x_{i}} \frac{D}{D t}\left(S_{i j}^{\prime \prime}\right)} \\
+\overline{\frac{\partial \alpha}{\partial c^{\prime \prime}} \frac{S_{i j}^{\prime \prime} \frac{D}{D t}\left(\frac{\partial c^{\prime \prime}}{\partial x_{i}}\right)}{\partial x_{j}}} .
\end{aligned}
$$

Using the definition of $S_{i j}^{\prime \prime}$ and some algebraic simplification leads to :

$$
\overline{\rho \frac{D}{D t}\left(\alpha \frac{\partial c^{\prime \prime}}{\partial x_{i}} S_{i j}^{\prime \prime} \frac{\partial c^{\prime \prime}}{\partial x_{j}}\right)}=\underbrace{2 \overline{\rho \alpha} \frac{\partial c^{\prime \prime}}{\partial x_{i}} S_{i j}^{\prime \prime} \frac{D}{D t}\left(\frac{\partial c^{\prime \prime}}{\partial x_{j}}\right)}_{\text {Term } 1}+\underbrace{\left.\overline{\rho \alpha} \frac{\left.\partial c^{\prime \prime} \frac{\partial c^{\prime \prime}}{\partial x_{j}} \frac{D}{\partial x_{i}} \frac{\partial u_{i}^{\prime \prime}}{\partial t}\right)}{\partial x_{j}}\right)}_{\text {Term } 2} .
$$

Term 1 and Term 2 in Eq. (15) can be simplified to give the final transport equation for $\widetilde{\Delta}_{c}$.

The details for the complete derivation are given in Appendix-A. After some algebra the transport equation for $\widetilde{\Delta}_{c}$ can be obtained as:

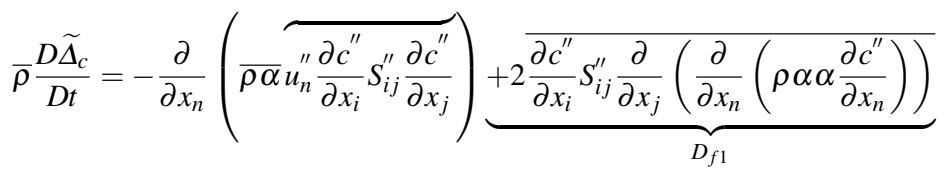

$$
\begin{aligned}
& \underbrace{\overline{+\alpha \frac{\partial c^{\prime \prime}}{\partial x_{i}} \frac{\partial c^{\prime \prime}}{\partial x_{j}} \frac{\partial}{\partial x_{n}}\left(\frac{\partial \tau_{i n}^{\prime \prime}}{\partial x_{j}}\right)}}_{D_{f 2}}+F_{1}+F_{2}+F_{3}+F_{4},
\end{aligned}
$$


where $D_{f 1}$ and $D_{f 2}$ represent diffusion/dissipation terms, $F_{1}$ represents source terms due to pressure gradient and reaction rate :

$$
F_{1}=\underbrace{\overline{2 \alpha \frac{\partial c^{\prime \prime}}{\partial x_{i}} S_{i j}^{\prime \prime} \frac{\partial \dot{\omega}_{c}^{\prime \prime}}{\partial x_{j}}}}_{F_{11}} \underbrace{-\overline{\alpha \frac{\partial c^{\prime \prime}}{\partial x_{i}} \frac{\partial c^{\prime \prime}}{\partial x_{j}} \frac{\partial}{\partial x_{i}}\left(\frac{\partial p^{\prime}}{\partial x_{j}}\right)}}_{F_{12}},
$$

$F_{2}$ represents terms arising due to turbulent transport :

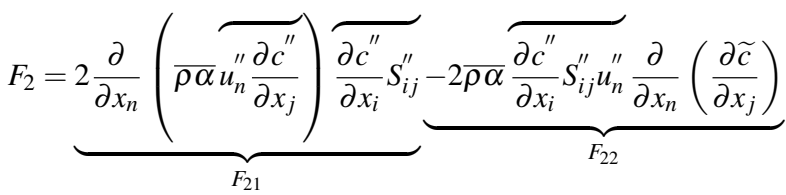

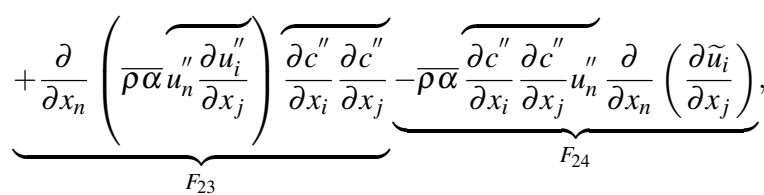

$F_{3}$ represents the dilatation terms:

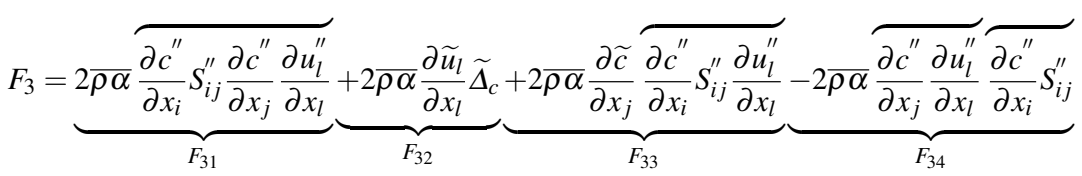

$$
\begin{aligned}
& \underbrace{+\alpha \frac{\partial \rho}{\partial x_{j}} \frac{\partial p^{\prime}}{\partial x_{i}} \frac{\partial c^{\prime \prime}}{\partial x_{j}} \frac{\partial c^{\prime \prime}}{\partial x_{i}} \frac{1}{\rho}}_{F_{35}} \underbrace{-\overline{\alpha \frac{\partial \rho}{\partial x_{j}} \frac{\partial \tau_{i n}^{\prime \prime}}{\partial x_{n}} \frac{\partial c^{\prime \prime}}{\partial x_{i}} \frac{\partial c^{\prime \prime}}{\partial x_{j}} \frac{1}{\rho}}}_{F_{36}},
\end{aligned}
$$

and $F_{4}$ represents turbulent straining terms :

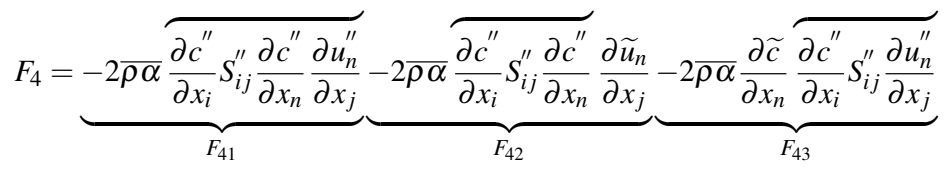

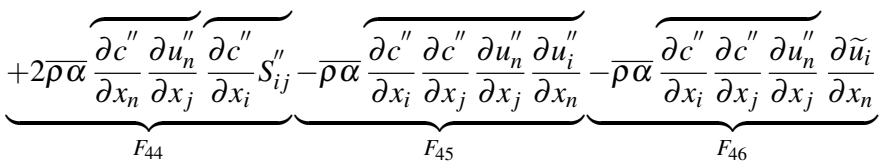

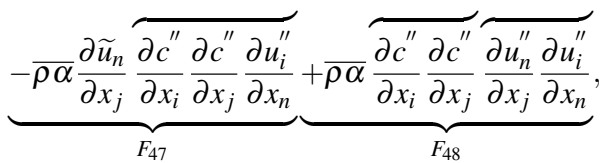




\section{Leading order terms in the $\widetilde{\Delta}_{c}$ evolution equation}

Repeating the order of magnitude analysis described in section 2 Eq. (16) leads to :

$$
D_{f}+F_{1}+F_{D}+F_{T S} \simeq 0,
$$

where $D_{f}$ represents the leading order diffusion/dissipation terms as :

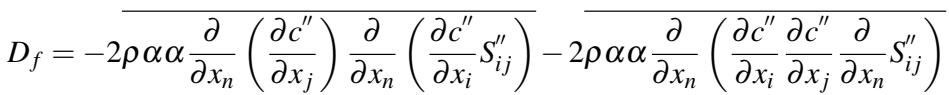

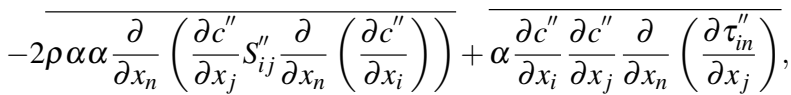

$F_{D}$ represents the leading order dilatation terms as:

$$
F_{D}=F_{31}+F_{34}+F_{35}+F_{36},
$$

and $F_{T S}$ represents the leading order turbulent straining terms as:

$$
F_{T S}=F_{41}+F_{44}+F_{45}+F_{48}
$$

Note that all the leading order terms in Eq. (16) scale as :

$$
\mathscr{O}\left(\rho_{u}\left(\frac{u_{L}^{0}}{\delta_{L}^{0}}\right)^{3} ; 1\right)
$$

A detailed order of magnitude analysis for all the terms in Eq. (16) is given in Appendix-B.

Here our interest is to close the leading order terms by suitable approximations. This is achieved via detailed interrogation of a Direct Numerical Simulation (DNS) data set of turbulent premixed V-flame in the corrugated flamelet regime. The details of the DNS are discussed in the next section. 


\section{Direct Numerical Simulation data}

In this study, the DNS data produced by Dunstan et al [21] for a turbulent premixed Vflame has been used. In the V-flame configuration, a stationary, non-planar flame is produced which is oblique to the mean flow, and subject to mean shear, strong tangential convection by the mean flow and flow divergence [20]. This type of configuration is characterised by a continuously developing flame in a statistically two dimensional mean flow field; the turbulence intensity along the leading edge of the flame brush decreases significantly while the individual flame elements are convected in the stream wise direction [20].

The V-flame in the DNS is representative of a lean, unit Lewis number flame with preheated reactants. The combustion kinetics are approximated by a single step reaction [21, 20]. DNS of the V-flame was carried out using a code called SENGA2, in which the conservation equations for mass, momentum, energy and reacting species are solved for compressible flow $[21,20,23,11,18]$. The spatial derivatives for interior grid points are calculated by using a 10th order central difference scheme, which gradually reduce to a 2 nd order scheme for all outflow boundaries, and 4th order scheme at the inlet boundary. The solution is evolved in time by using a 4th order explicit Runge-Kutta scheme. The transport coefficients have a temperature dependence approximated by 5 th order polynomials following the CHEMKIN formats. Navier-Stokes Characteristic Boundary Conditions (NSCBC) have been applied to all non-periodic boundaries. The standard NSCBC has been modified to accommodate the steep thermal and compositional gradients when the flame crosses the boundary. This has been done to avoid large pressure perturbations which can have a significant influence on the interior solution. Further details on the boundary conditions can be found in $[21,31,32,34,37]$. Similar DNS data sets have been used in several modelling stud- 
ies for scalar dissipation rate, turbulent flame speed and flame generated noise in premixed turbulent combustion $[21,20,19,26]$.

The domain for the V-flame simulation is a cube of side $29.69 \delta_{L}^{0}$. The domain is discretised by a $512 \times 512 \times 512$ node uniform grid, ensuring a minimum resolution of about 10 grid points is maintained to resolve the laminar flame thermal thickness $\delta_{L}^{0}$. The flame is stabilised by a flame holder positioned at $3.49 \delta_{L}^{0}$ from the inlet plane; this is achieved by fixing the mass fraction through a Gaussian weighting function and restricting velocities to their mean values (further details on the flame holder can be found in $[21,20])$. The schematic of the computational domain is given in figure 1. The simulation is run for one flow through, to allow for the transients to decay before collecting data for analysis.

The global thermochemical parameters used in the DNS are; planar, unstretched laminar flame speed $u_{L}^{0}=0.6034 \mathrm{~m} / \mathrm{s}$; laminar flame thermal thickness $\delta_{L}^{0}=\left(T_{a d}-T_{0}\right) / \max |\nabla T|=$ $0.43 \mathrm{~mm}$ (where $T_{a d}=2113.3 \mathrm{~K}$ is the adiabatic flame temperature and $T_{0}=600.0 \mathrm{~K}$ is the inlet reactant temperature); heat release parameter $\tau=\left(T_{a d}-T_{0}\right) / T_{0}=2.52$; Zeldovitch number $\beta=7.13$; characteristic laminar flame time is given by $\tau_{f}=\delta_{L}^{0} / u_{L}^{0}=0.71 \mathrm{~ms}$ and the laminar diffusive thickness $\delta_{L}=\alpha / u_{L}^{0}=0.1207 \mathrm{~mm}$. For the purpose of comparison with real air-fuel mixture flames, the Zeldovitch number and the heat release parameter are representative of a pre-heated premixed methane-air flame with an equivalence ratio $\phi \approx 0.6$ [21].

The values of the inlet turbulent Reynolds number $\left(R e_{l_{t}}\right)$, Karlovitz number $(K a)$ and Damköhler number $(\mathrm{Da})$ based on the laminar flame diffusive thickness and inlet flow conditions are summarised in table 1 , where $u_{\text {in }}^{\prime}$ is the rms velocity at the inlet, $\bar{u}_{\text {in }}$ is the mean inlet velocity in the $y$-direction as shown in figure $1, v$ is the kinematic viscosity and $l_{t}$ is the integral length scale. 
Figure 2 show the contours of $0.1 \leq \tilde{c} \leq 0.9$ in the V-flame DNS, where the $x^{+}$and $y^{+}$ represent the directions normalised by the thermal thickness of the the flame $\delta_{L}^{0}$.

\subsection{Post-processing and numerical resolution}

During the post processing of the DNS, the data was averaged in time over 21 instantaneous realisations of the flow (over a total sample time corresponding to one flow through), and space averaged in the periodic $(z)$ direction. Spatial derivatives of fluctuating quantities required by the terms in Eq. (21) are calculated using the same numerical algorithm as used in the DNS. The present analysis is restricted to $y^{+}>20$, beyond which the flame has sufficient time to develop after ignition at the flame holder [21,19]. Three sampling locations downstream of the flame holder at $y^{+} \approx 23, y^{+} \approx 25$ and $y^{+} \approx 27$ are used, labelled as a, $\mathrm{b}$, and $\mathrm{c}$ in figure 2. The flame is influenced by the level of turbulence upstream of the flame, hence non-dimensional parameters such as $K a, D a$ and $R e_{l_{t}}$ are reported for all the sampling locations at $\widetilde{c} \approx 0.001$ in table 2 . It can be observed in table 2 that the flame lies in the corrugated flamelet regime, and $R e_{l_{t}}$ remains close to approximately 20 at all the sampling locations used for this study. All the results have been normalised using $\rho_{u}, u_{L}^{0}$ and $\delta_{L}^{0}$. As the thickness of $\widetilde{c}$ changes along the mean flow direction and $\widetilde{c}$ varies monotonically from the flame centreline, $\widetilde{c}$ is used to denote the location inside the flame brush in the results discussed below.

As mentioned earlier a resolution of minimum 10 grid points is used to resolve the laminar flame thickness; in the regions where the data is extracted, the number of grid points in the flame structure have increased to almost 30. This can be noted in figure 3 where the different time realisations for the instantaneous progress variable are shown for all sampling locations. This level of spatial resolution is assumed to be sufficient for the evaluation of the 
higher order (i.e. 4 th or 5 th order) correlations with high order derivatives of the fluctuating quantities. Sufficiency can be further established by the numerical resolution criterion of Poinsot et al [29]:

$$
\operatorname{Re}_{l_{t}} D a<2(1+\tau)^{0.7}\left(\frac{N_{x}}{n_{g}} n_{e}\right)^{2}
$$

where $N_{x}$ represents the number of grid points in the direction of flame gradients, $n_{g}$ represents the number of grid points in the flame structure and $n_{e}$ represents the number of large scale eddies in the domain. The values of $n_{e}$ have been calculated using the turbulence characteristics upstream of the flame as reported in table 2. Table 3 shows the results obtained by using Eq. (25) for all sampling locations; the inequality in Eq. (25) is satisfied for all the sampling locations.

The statistical convergence of the Reynolds/Favre averaged quantities is tested by comparing the average data from all 21 realisations against the average data from 10,12,15 and 18 realisations. The time evolution of $D_{f}$ (one of the highest moments with the highest order of derivatives in Eq. (22)) is shown in figure 4 for all sampling locations. Statistical convergence has been achieved for $D_{f}$ after 15 averaged realisations. Sample size of 21 instantaneous realisations is thus deemed sufficient for calculating the leading order terms in the flame turbulence interaction transport equation.

\section{Results and discussion}

\subsection{Eigenvector analysis and alignment statistics}

The behaviour of the flame turbulence interaction relies on the alignment of the strain rate eigenvectors with the scalar gradient, hence an eigenvector analysis has been undertaken for the V-flame DNS. 
The eigenvectors for the strain rate have been calculated by using LAPACK libraries for FORTRAN [1]. Figures 5, 6 and 7 show the pdfs of direction cosines between the strain rate eigenvectors and the flame gradient at $y^{+} \approx 23, y^{+} \approx 25$ and $y^{+} \approx 27$ respectively for different iso-surfaces of $c$. It can be observed in figures 5(a), 6(a) and 7(a) that the most probable inner product tending towards unity is the inner product between $e_{\alpha}$ and $\nabla c$, which implies that the extensive strain rate eigenvector aligns preferentially with the flame gradient for $0.3 \leq c \leq 0.7$ at all the sampling locations in the DNS. It should be noted here however that the direct interpretation of strain rate orientation from direction cosines can be obscured by the non-linearity of the cosine function. In case of a uniformly distributed angle between two vectors, the pdf of $\cos \theta$ shows higher probabilities of unity [33], and caution must be taken when interpreting alignment pdfs based on direction cosines.

Steinberg et al [33] argue that the actual physical orientation of the strain rate field with the flame gradient can be better represented by the pdfs of the angle between the two vectors directly. Figures 5(b), 6(b) and 7(b) show the pdfs of the angles between the extensive strain rate eigenvector and the flame gradient at $y^{+} \approx 23, y^{+} \approx 25$ and $y^{+} \approx 27$ respectively for different iso-surfaces of $c$. It can be observed that the most probable alignment between $e_{\alpha}$ and $\nabla c$ occurs at $0 \leq \theta_{\alpha} \leq 0.5$ for $0.3 \leq c \leq 0.7$ at all sampling locations, which again implies that $e_{\alpha}$ preferentially aligns with $\nabla c$ for $0.3 \leq c \leq 0.7$. This is in agreement with the earlier studies $[15,22]$ as the chemical reactions releasing heat compete with the local fluid dynamic processes, thus causing the flame gradient to align with the most extensive strain rate eigenvector.

The non-linearity induced by the cosine function can be noticed by comparing the pdfs of direction cosines between $e_{\alpha}$ and $\nabla c$ and the pdfs for the resulting angles between $e_{\alpha}$ and $\nabla c$. The pdfs for the direction cosines between $e_{\alpha}$ and $\nabla c$ show a bias towards higher prob- 
ability of unity as shown in figures 5(a), 6(a) and 7(a); whereas the pdfs for the associated angles show a range of angles with lower probability as shown in figures 5(b), 6(b) and 7(b).

Note that $e_{\alpha}$ and $\nabla c$ are not completely aligned for $c=0.1$ and $c=0.9$ iso-surfaces at all sampling locations. The dilatation due to heat release is low near $c=0.1$ and $c=0.9$ iso-surfaces, as shown in figure 8 for all the sampling locations in the V-flame. The mean shear overcomes the dilatation effects and enters the flame structure at $c=0.1$ and $c=0.9$, thus causing the flame gradients to move away from the extensive strain rate eigenvector and towards the compressive strain rate eigenvector as shown in figures 5(b), 6(b), 7(b), 5(f), 6(f) and 7(f). This phenomenon has been noted in the earlier studies by Minamoto et al [27] in case of reacting flows and by Ashurst et al [2] in case of non reacting flows. These changes in alignment characteristics across the flame structure signify the importance of the transport equation for $\widetilde{\Delta}_{c}$, as the transport equation allows for a more flexible approach to incorporate the correct physics and flow history effects into the modelling strategy.

5.2 Leading order terms in the $\widetilde{\Delta}_{c}$ evolution equation

Figures 9(a) - 9(c) show the profiles for the leading order terms in the $\widetilde{\Delta}_{c}$ transport equation at $y^{+} \approx 23, y^{+} \approx 25$ and $y^{+} \approx 27$. The over all behaviour of $\widetilde{\Delta}_{c}$ transport equation is strongly controlled by a competition between the source terms $\left(F_{1}\right)$, diffusion/dissipation process $\left(D_{f}\right)$, turbulent strain rate $\left(F_{T S}\right)$ and the dilatation rate $\left(F_{D}\right)$ as shown in figure 9.

It can be seen in figure 10 that there is a competition between the turbulent strain rate $\left(F_{T S}\right)$ and the dilatation rate $\left(F_{D}\right)$ at all the sampling locations, which is in agreement with theories proposed in earlier studies $[13,36]$. These earlier studies further suggest that the competition between $F_{T S}$ and $F_{D}$ significantly influences the evolution of $\widetilde{\Delta}_{C}$ and is considered to be an important phenomenon $[13,36]$. We argue here that the source terms $\left(F_{1}\right)$ and 
diffusion/dissipation processes $\left(D_{f}\right)$ are the main contributing terms in the evolution of $\widetilde{\Delta}_{c}$ as shown in figure 9.

\subsection{Model for $F_{T S}$}

Expanding the expression for $F_{T S}$ in Eq. (24) leads to :

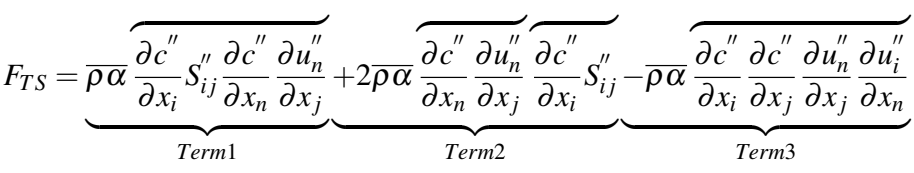

$$
\begin{aligned}
& \underbrace{+\overline{\rho \alpha} \overbrace{\frac{\partial c^{\prime \prime}}{\partial x_{i}} \frac{\partial c^{\prime \prime}}{\partial x_{j}}} \overbrace{\frac{\partial u_{n}^{\prime \prime}}{\partial x_{j}} \frac{\partial u_{i}^{\prime \prime}}{\partial x_{n}}}}_{T^{\prime}}
\end{aligned}
$$

All the terms in Eq. (26) require closures. The scalar gradient and strain rate contribution in

term 1, Eq. (26) can be scaled as :

$$
\rho \alpha \frac{\partial c^{\prime \prime}}{\partial x_{i}} S_{i j}^{\prime \prime} \frac{\partial c^{\prime \prime}}{\partial x_{n}} \sim \bar{\rho} \widetilde{\Delta}_{c}
$$

and the turbulent straining represented by the fluctuating velocity gradients can be scaled by a turbulent time scale as :

$$
\frac{\partial u_{i}^{\prime \prime}}{\partial x_{j}} \sim \frac{\widetilde{\varepsilon}}{\widetilde{k}} .
$$

Similar scaling arguments are used to scale the rest of the terms in Eq. (26), thus leading to $:$

$$
F_{T S} \approx C_{b} \bar{\rho} \underset{\tilde{k}}{\widetilde{\varepsilon}} \widetilde{\Delta_{c}}
$$

where $C_{b}$ is a scaling factor for the model.

The value of $C_{b}$ is of key importance. Following earlier modelling strategies used in the $\widetilde{\varepsilon}_{c}$ transport equation $[16,14,17]$ a functional form of $C_{b}$ is proposed as :

$$
C_{b}=-\left[3.5+\frac{0.01 \operatorname{erf}\left(K a_{L}\right)}{0.01+\sqrt{\operatorname{erf}\left(\operatorname{Re}_{l_{t}}\right)}}\right],
$$


where $R e_{l_{t}}$ is defined as [28]:

$$
\operatorname{Re}_{l_{t}}=\frac{u^{\prime} l_{t}}{v_{u}}
$$

and $K a_{L}$ is defined as [28]:

$$
K a_{L}=\left(u^{\prime} / u_{L}^{0}\right)^{3 / 2}\left(\delta_{L} / l_{t}\right)^{1 / 2}
$$

$u^{\prime}$ and $l_{t}$ in Eq (29) and Eq (30) are defined as :

$$
u^{\prime}=\sqrt{\frac{2 \widetilde{k}}{3}} \text { and } l_{t}=\frac{u^{\prime 3}}{\widetilde{\varepsilon}} \text {. }
$$

The function for $C_{b}$ is one of several possible empirical relations which lead to a physically plausible result. It should be noted that the turbulent strain rate increases with an increase in small scale turbulence represented by Karlovitz number; this phenomenon is captured by the functional form of $C_{b}$ presented in Eq. (28). The function in Eq. (28) reaches an asymptotic value as $u^{\prime} \rightarrow \infty$. Similar scaling factors have been proposed by Chakraborty and Swaminathan [17] for the closures of the $\widetilde{\varepsilon}_{c}$ transport equation.

Comparisons of the model against the DNS data set at all the sampling locations are given in figure 11. Throughout, the model performance improves as the distance of the sampling location from the flame holder increases. The scaling factor $C_{b}$ in Eq. (28) is only valid for high Damköhler number flames, and further tests need to be carried out to check the validity of the model for different combustion conditions.

\subsection{Model for $F_{D}$}

It can be observed in Eq. (23) that : 


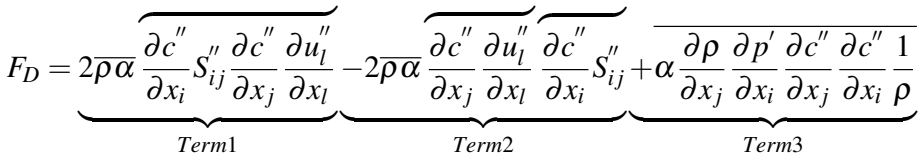

$$
\begin{aligned}
& \underbrace{-\alpha \frac{\partial \rho}{\partial x_{j}} \frac{\partial \tau_{i n}^{\prime \prime}}{\partial x_{n}} \frac{\partial c^{\prime \prime}}{\partial x_{i}} \frac{\partial c^{\prime \prime}}{\partial x_{j}} \frac{1}{\rho}}_{\text {Term } 4} .
\end{aligned}
$$

We propose that the contributions involving the scalar gradients and the strain rate in term 1

Eq. (32) scale as :

$$
\rho \alpha \frac{\partial c^{\prime \prime}}{\partial x_{i}} S_{i j}^{\prime \prime} \frac{\partial c^{\prime \prime}}{\partial x_{j}} \backsim \bar{\rho} \widetilde{\Delta}_{c},
$$

while the contributions involving the dilatation represented by the divergence of the velocity field scale as :

$$
\frac{\partial u_{l}^{\prime \prime}}{\partial x_{l}} \sim \tau \frac{u_{L}^{0}}{\delta_{L}}
$$

Similar scaling arguments apply to term 2 in Eq. (32). Term 3 in Eq. (32) includes the effects of pressure gradient which is a source term for the transport of momentum and thus containing the effects of the strain rate; the density gradients include the effects of dilatation, and hence combining the two observations term 3 in Eq. (32) scales as :

$$
\alpha \frac{\partial \rho}{\partial x_{j}} \frac{\partial p^{\prime}}{\partial x_{i}} \frac{\partial c^{\prime \prime}}{\partial x_{j}} \frac{\partial c^{\prime \prime}}{\partial x_{i}} \frac{1}{\rho} \sim \bar{\rho} \widetilde{\Delta}_{c} \tau \frac{u_{L}^{0}}{\delta_{L}} .
$$

Term 4 in Eq. (32) can be simplified by substituting $\tau_{\text {in }}^{\prime \prime}$ as $\tau_{i n}^{\prime \prime} \approx 2 \mu S_{i n}^{\prime \prime}$, where $\mu \approx \rho \alpha$, and assuming that the density gradients associated with $\tau_{i n}^{\prime \prime}$ are small, thus leading to :

$$
-\overline{\rho \alpha \alpha \frac{\partial \rho}{\partial x_{j}} \frac{\partial S_{i n}^{\prime \prime}}{\partial x_{n}} \frac{\partial c^{\prime \prime}}{\partial x_{j}} \frac{\partial c^{\prime \prime}}{\partial x_{i}} \frac{1}{\rho} .}
$$

Following the earlier scaling arguments the expression in Eq. (33) scales as :

$$
\sim \bar{\rho} \widetilde{\Delta}_{c} \tau \frac{u_{L}^{0}}{\delta_{L}}
$$

Thus leading to the model :

$$
F_{D} \approx C_{c} D a_{L} \bar{\rho} \widetilde{\Delta}_{c} \tau \frac{u_{L}^{0}}{\delta_{L}}
$$


where $D a_{L}$ represents the local Damköhler number and is defined as :

$$
D a_{L}=\frac{\left(u_{L}^{0} / \delta_{L}\right)}{(\widetilde{\varepsilon} / \widetilde{k})},
$$

and $C_{c}$ is a scaling factor for the model. Note that an expression for the local Damköhler number has been proposed by Chakraborty and Swaminathan [16], but their definition is based on the laminar flame thermal thickness $\delta_{L}^{0}$ where as the definition in Eq. (35) is based on the laminar flame diffusive thickness $\delta_{L}$. The model proposed in Eq. (34) has an explicit dependence on the heat release parameter $\tau$ and the local Damköhler number $D a_{L}$, thus allowing the model to account for the changes in heat release and turbulence and their influence on dilatation in the $\widetilde{\Delta}_{c}$ evolution. It can be observed in Eq. (34) that in the limiting case of $u^{\prime} \rightarrow \infty$ and $D a \rightarrow 0$ the influence of dilatation vanishes. Furthermore an explicit dependence of the model on $\tau$ allows the model to vanishes in the limiting case of cold flow turbulence.

The accuracy of the model in Eq. (34) relies on the choice of the scaling factor $C_{c}$; here we include the effects of turbulent Reynolds number into our modelling strategy and define $C_{c}$ as :

$$
C_{c}=\frac{0.01+3.5 \operatorname{erf}\left(K a_{L}\right)}{13 \operatorname{erf}\left(\left(\operatorname{Re}_{l_{t}}^{3} / 17.5\right)+0.01\right)}
$$

Note that $C_{c}$ decreases as $u^{\prime}$ increases, eventually reducing to an asymptotic value as $R e_{l_{t}} \rightarrow$ $\infty$ and $K a_{L} \rightarrow \infty$. This has been done because the dilatation rate decreases with increasing $u^{\prime}$ values. The Karlovitz number effects have been included in Eq. (36) to account for the changes in the dilatation rate due to small scale turbulence. Furthermore in case of cold flows where $K a_{L} \rightarrow 0$ the function in Eq. (36) reduces to a very small number along with the contributions from the actual model in Eq. (34) going to zero. The results from the model are in good agreement with the DNS data as shown in in figure 12. The scaling factor $C_{c}$ 
presented in Eq. (36) is one of many possible empirical functions; and is only valid for

$D a>1$ and $K a<1$ flames. More DNS data in the thin reaction zone regime is needed to extend the modelling further and obtain more robust scaling factors.

5.5 Model for diffusion $D_{f}$ and $F_{1}$ source terms

$$
\begin{gathered}
D_{f}+F_{1}=-\overline{-2 \rho \alpha \alpha \frac{\partial}{\partial x_{n}}\left(\frac{\partial c^{\prime \prime}}{\partial x_{j}}\right) \frac{\partial}{\partial x_{n}}\left(\frac{\partial c^{\prime \prime}}{\partial x_{i}} S_{i j}^{\prime \prime}\right)}-\frac{\left.\overline{\rho \alpha \alpha \frac{\partial}{\partial x_{n}}\left(\frac{\partial c^{\prime \prime}}{\partial x_{i}} \frac{\partial c^{\prime \prime}}{\partial x_{j}}\right.} \frac{\partial}{\partial x_{n}} S_{i j}^{\prime \prime}\right)}{-2 \rho \alpha \alpha \frac{\partial}{\partial x_{n}}\left(\frac{\partial c^{\prime \prime}}{\partial x_{j}} S_{i j}^{\prime \prime} \frac{\partial}{\partial x_{n}}\left(\frac{\partial c^{\prime \prime}}{\partial x_{i}}\right)\right)}+\overline{\alpha \frac{\partial c^{\prime \prime}}{\partial x_{i}} \frac{\partial c^{\prime \prime}}{\partial x_{j}} \frac{\partial}{\partial x_{n}}\left(\frac{\partial \tau_{i n}^{\prime \prime}}{\partial x_{j}}\right)} \\
+2 \alpha \frac{\partial c^{\prime \prime}}{\partial x_{i}} S_{i j}^{\prime \prime} \frac{\partial \dot{\omega}_{c}^{\prime \prime}}{\partial x_{j}}-\alpha \frac{\partial c^{\prime \prime}}{\partial x_{i}} \frac{\partial c^{\prime \prime}}{\partial x_{j}} \frac{\partial}{\partial x_{i}}\left(\frac{\partial p^{\prime}}{\partial x_{j}}\right) .
\end{gathered}
$$

It can be seen in figure 9 that term $F_{1}$ is dominantly a source and $D_{f}$ is dominantly a sink for $\widetilde{\Delta}_{c}$ evolution. The combined terms in Eq. (37) scale as a product of flame normal strain rate $\widetilde{\Delta}_{c} / \widetilde{\varepsilon}_{c}$ and flame turbulence interaction $\widetilde{\Delta}_{c}$ as :

$$
\sim \widetilde{\Delta}_{c}^{2} / \widetilde{\varepsilon}_{c}
$$

thus leading to :

$$
D_{f}+F_{1} \approx-C_{a} \bar{\rho} \frac{\widetilde{\Delta}_{c}^{2}}{\widetilde{\varepsilon}_{c}} .
$$

$C_{a}$ is a scaling factor in Eq. (38), and the negative sign is used due to the (over all) sink nature of $D_{f}+F_{1}$ as shown in figure 13 . The sum of terms $D_{f}$ and $F_{1}$ has a dominant effect in $\widetilde{\Delta}_{c}$ evolution, thus the ratio $\widetilde{\Delta}_{c}^{2} / \widetilde{\varepsilon}_{c}$ in Eq. (38) represents the rate of change of flame turbulence interaction, $\widetilde{\Delta}_{c}^{2} / \widetilde{\varepsilon}_{c} \approx d \widetilde{\Delta}_{c} / d t$.

The value of $C_{a}$ in Eq. (38) is a matter of calibration and is dependent on the DNS data set used for model calibration. Here we propose a value of $C_{a}$ based on the ratio of turbulent Reynolds number and local Karlovitz number, thus accounting for the changes in the local flow conditions : 


$$
C_{a}=\left[4+\frac{1.9 \operatorname{erf}\left(\operatorname{Re}_{l_{t}}\right)}{\operatorname{erf}\left(\left(K a_{L}+1\right) / 50\right)}\right]
$$

This has been done because the flame normal strain rate decreases with increasing strength of the small scale turbulence represented by Karlovitz numbers [15]. It can be seen in Eq. (39) that the value of $C_{a}$ reaches an asymptotic value as $R e_{l_{t}} \rightarrow \infty$ and $K a_{L} \rightarrow \infty$. Note that the exact behaviour of $D_{f}+F_{1}$ at higher $R e_{l_{t}}$ and $K a$ conditions is not known and more DNS data in the thin reaction zone regime is needed to extend the modelling further. The model proposed for $D_{f}+F_{1}$ are in qualitative agreement with the DNS data as shown in figure 13 .

\section{Summary and Conclusions}

Flame turbulence interaction is an important quantity in turbulent premixed combustion modelling. It has been shown in previous studies that the effect of strain rate on the transport of scalar dissipation is dominated by the interaction between the fluctuating scalar gradients and the fluctuating strain rate (here denoted by $\widetilde{\Delta}_{c}$ ). An accurate representation of the flame turbulence interaction can be obtained from the leading order terms in the evolution equation

for $\widetilde{\Delta}_{c}$. In this paper an evolution equation for $\widetilde{\Delta}_{c}$ has been derived and an order of magnitude analysis under the joint assumption of high Reynolds and Damköhler numbers has been done to identify the leading order terms. The leading order terms have been analysed via the DNS results of Dunstan et al [21]. It has been found that the turbulent strain rate and the dilatation rate compete in $\widetilde{\Delta}_{c}$ evolution, which is in agreement with the theories proposed in earlier studies $[13,36]$. It has also been found that there is also competition between the source terms (pressure gradient and reaction rate) and the diffusion/dissipation processes. It is argued in this study that the overall behaviour of $\widetilde{\Delta}_{c}$ evolution equation is determined by the competition between source and diffusion/dissipation terms. 
Closures for the leading order terms have been proposed and compared against the DNS data set at different locations. The comparisons of modelled predictions and the DNS values are in good agreement for the combustion conditions considered in this study. In the light of a recent study by Chakarborty and Swaminathan [17] the closures for the leading order terms have been made functions of turbulent Reynolds and local Karlovitz numbers. The present modelling strategy is only able to capture the flame turbulence interaction in the wrinkled/corrugated flamelet regime $(D a>1$ and $K a<1)$. More investigations of DNS data sets with different combustion conditions and turbulent Reynolds number are needed to fully understand the behaviour of the leading order terms in $\widetilde{\Delta}_{c}$ evolution equation, which would lead to more robust modelling strategies applicable to a wide range of combustion conditions. This forms part of the on going work.

Acknowledgements The authors would like to thank Dr T.D. Dunstan and Professor N. Swaminathan for making the DNS data set available.

\section{Appendix-A}

In order to resolve Term 1 in Eq. (14), a transport equation for the gradient of $c^{\prime \prime}$ is required. That is achieved by using the definition $c^{\prime \prime}=c-\widetilde{c}$ and taking the derivative of the instantaneous progress variable transport equation to write :

$$
\begin{gathered}
\rho \alpha \frac{\partial}{\partial t}\left(\frac{\partial c}{\partial x_{j}}\right)+\rho \alpha u_{n} \frac{\partial}{\partial x_{n}}\left(\frac{\partial c}{\partial x_{j}}\right)=\alpha \frac{\partial}{\partial x_{j}}\left(\frac{\partial}{\partial x_{n}}\left(\rho \alpha \frac{\partial c}{\partial x_{n}}\right)\right)+\frac{\partial \dot{\omega}_{c}}{\partial x_{j}} \\
-\rho \alpha \frac{\partial c}{\partial x_{n}} \frac{\partial u_{n}}{\partial x_{j}}+\rho \alpha \frac{\partial c}{\partial x_{j}}\left(\frac{\partial u_{l}}{\partial x_{l}}\right)
\end{gathered}
$$


Applying the Reynolds decomposition and Favre averaging Eq. (40) leads to:

$$
\begin{gathered}
\overline{\rho \alpha} \frac{\partial}{\partial t}\left(\frac{\partial \widetilde{c}}{\partial x_{j}}\right)+\overline{\rho \alpha} \widetilde{u}_{n} \frac{\partial}{\partial x_{n}}\left(\frac{\partial \widetilde{c}}{\partial x_{j}}\right)=\frac{\partial}{\partial x_{j}}\left(\frac{\partial}{\partial x_{n}}\left(\overline{\rho \alpha \alpha} \frac{\partial \widetilde{c}}{\partial x_{n}}\right)\right)-\frac{\partial}{\partial x_{n}}\left(\overline{\rho \alpha} \bar{u}_{n}^{\prime \prime} \frac{\partial c^{\prime \prime}}{\partial x_{j}}\right) \\
+\bar{\alpha} \frac{\partial}{\partial x_{j}} \overline{\dot{\omega}_{c}}+\rho \alpha \frac{\partial c^{\prime \prime}}{\partial x_{j}} \frac{\partial u_{l}^{\prime \prime}}{\partial x_{l}}+\overline{\rho \alpha} \frac{\partial \widetilde{c}}{\partial x_{j}} \frac{\partial \widetilde{u}_{l}}{\partial x_{l}}-\overline{\rho \alpha \frac{\partial c^{\prime \prime}}{\partial x_{n}} \frac{\partial u_{n}^{\prime \prime}}{\partial x_{j}}}+\overline{\rho \alpha} \frac{\partial \widetilde{c}}{\partial x_{n}} \frac{\partial \widetilde{u}_{n}}{\partial x_{j}}
\end{gathered}
$$

Subtracting the mean equation from the instantaneous equation and then multiplying the resulting equation with $\frac{\partial c^{\prime \prime}}{\partial x_{i}} S_{i j}^{\prime \prime}$ and Favre averaging leads to:

$$
\begin{aligned}
& \overline{\rho \alpha} \overbrace{\frac{\partial c^{\prime \prime}}{\partial x_{i}} S_{i j}^{\prime \prime} \frac{D}{D t}\left(\frac{\partial c^{\prime \prime}}{\partial x_{j}}\right)}=\overline{\frac{\partial c^{\prime \prime}}{\partial x_{i}} S_{i j}^{\prime \prime} \frac{\partial}{\partial x_{j}}\left(\frac{\partial}{\partial x_{n}}\left(\rho \alpha \alpha \frac{\partial c^{\prime \prime}}{\partial x_{n}}\right)\right)}
\end{aligned}
$$

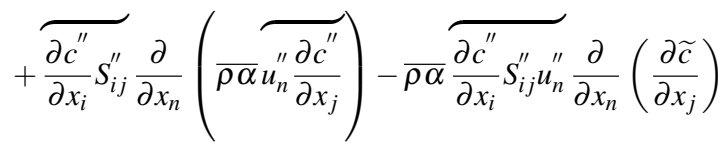

$$
\begin{aligned}
& +\overline{\alpha \frac{\partial c^{\prime \prime}}{\partial x_{i}} S_{i j}^{\prime \prime} \frac{\partial}{\partial x_{j}} \dot{\omega}_{c}^{\prime \prime}}+\overline{\rho \alpha} \overline{\frac{\partial c^{\prime \prime}}{\partial x_{i}} S_{i j}^{\prime \prime} \frac{\partial c^{\prime \prime}}{\partial x_{j}} \frac{\partial u_{l}^{\prime \prime}}{\partial x_{l}}}+\overline{\rho \alpha} \frac{\partial \widetilde{u}_{l}}{\partial x_{l}} \overbrace{\frac{\partial c^{\prime \prime}}{\partial x_{i}} S_{i j}^{\prime \prime} \frac{\partial c^{\prime \prime}}{\partial x_{j}}} \\
& +\overline{\rho \alpha} \frac{\partial \widetilde{c}}{\partial x_{j}} \overbrace{\frac{\partial c^{\prime \prime}}{\partial x_{i}} S_{i j}^{\prime \prime} \frac{\partial u_{l}^{\prime \prime}}{\partial x_{l}}}-\overline{\rho \alpha} \overbrace{\frac{\partial c^{\prime \prime}}{\partial x_{j}} \frac{\partial u_{l}^{\prime \prime}}{\partial x_{l}}} \overbrace{\frac{\partial c^{\prime \prime}}{\partial x_{i}} S_{i j}^{\prime \prime}}
\end{aligned}
$$

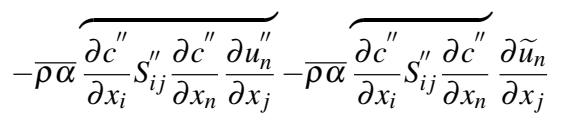

$$
\begin{aligned}
& -\overline{\rho \alpha} \frac{\partial \widetilde{c}}{\partial x_{n}} \overbrace{\frac{\partial c^{\prime \prime}}{\partial x_{i}} S_{i j}^{\prime \prime} \frac{\partial u_{n}^{\prime \prime}}{\partial x_{j}}+\overline{\rho \alpha}} \overbrace{\frac{\partial c^{\prime \prime}}{\partial x_{n}} \frac{\partial u_{n}^{\prime \prime}}{\partial x_{j}}}^{\frac{\partial c^{\prime \prime}}{\partial x_{i}} S_{i j}^{\prime \prime}} .
\end{aligned}
$$

Term 2 in Eq. (14) can be obtained by using the transport equation for the gradient of $u_{i}^{\prime \prime}$. Using a similar procedure to above and taking the derivative of the instantaneous momentum transport equation to write:

$$
\begin{aligned}
\rho \alpha \frac{\partial}{\partial t}\left(\frac{\partial u_{i}}{\partial x_{j}}\right) & +\rho \alpha u_{n} \frac{\partial}{\partial x_{n}}\left(\frac{\partial u_{i}}{\partial x_{j}}\right)=-\alpha \frac{\partial}{\partial x_{i}}\left(\frac{\partial p}{\partial x_{j}}\right)+\alpha \frac{\partial}{\partial x_{n}}\left(\frac{\partial \tau_{i n}}{\partial x_{j}}\right) \\
& -\rho \alpha \frac{\partial u_{n}}{\partial x_{j}} \frac{\partial u_{i}}{\partial x_{n}}-\alpha \frac{\partial \rho}{\partial x_{j}}\left(-\frac{\partial p}{\partial x_{i}}+\frac{\partial \tau_{i n}}{\partial x_{n}}\right) \frac{1}{\rho}
\end{aligned}
$$

Applying the Reynolds decomposition and Favre averaging Eq. (43) leads to: 


$$
\begin{aligned}
& \overline{\rho \alpha} \frac{\partial}{\partial t}\left(\frac{\partial \widetilde{u}_{i}}{\partial x_{j}}\right)+\overline{\rho \alpha} \widetilde{u}_{n} \frac{\partial}{\partial x_{n}}\left(\frac{\partial \widetilde{u}_{i}}{\partial x_{j}}\right)=-\bar{\alpha} \frac{\partial}{\partial x_{i}}\left(\frac{\partial \bar{p}}{\partial x_{j}}\right)-\frac{\partial}{\partial x_{n}}\left(\widetilde{\rho \alpha u_{n}^{\prime \prime} \frac{\partial u_{i}^{\prime \prime}}{\partial x_{j}}}\right) \\
& +\bar{\alpha} \frac{\partial}{\partial x_{n}}\left(\frac{\partial \overline{\tau_{i n}}}{\partial x_{j}}\right)-\overline{\rho \alpha} \frac{\partial u_{n}^{\prime \prime}}{\partial x_{j}} \frac{\partial u_{i}^{\prime \prime}}{\partial x_{n}}-\overline{\rho \alpha} \frac{\partial \widetilde{u}_{n}}{\partial x_{j}} \frac{\partial \widetilde{u}_{i}}{\partial x_{n}}+\overline{\frac{\alpha}{\rho} \frac{\partial \rho}{\partial x_{j}}} \frac{\partial \bar{p}}{\partial x_{i}}-\bar{\alpha} \frac{\partial \rho}{\rho} \frac{\partial \overline{\tau_{i n}}}{\partial x_{n}} .
\end{aligned}
$$

Subtracting Eq. (44) from Eq. (43) then multiplying the resulting equation with $\frac{\partial c^{\prime \prime}}{\partial x_{i}} \frac{\partial c^{\prime \prime}}{\partial x_{j}}$ and Favre averaging leads to:

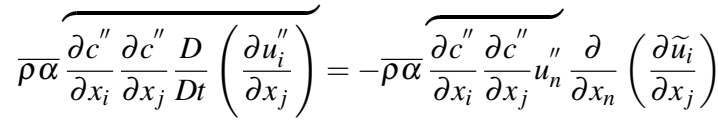

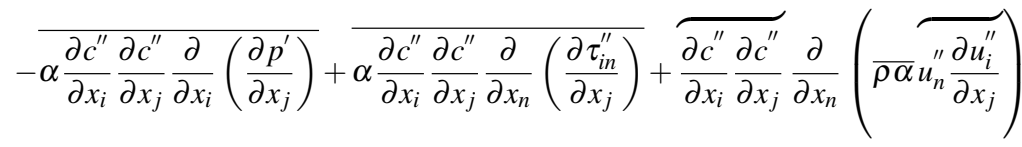

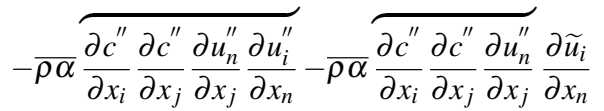

$$
\begin{aligned}
& -\overline{\rho \alpha} \frac{\partial \widetilde{u}_{n}}{\partial x_{j}} \overbrace{\frac{\partial c^{\prime \prime}}{\partial x_{j}} \frac{\partial c^{\prime \prime}}{\partial x_{i}} \frac{\partial u_{i}^{\prime \prime}}{\partial x_{n}}+\overline{\rho \alpha}} \overbrace{\frac{\partial c^{\prime \prime}}{\partial x_{i}} \frac{\partial c^{\prime \prime}}{\partial x_{j}}}^{\frac{\partial u_{n}^{\prime \prime}}{\partial x_{j}} \frac{\partial u_{i}^{\prime \prime}}{\partial x_{n}}}
\end{aligned}
$$

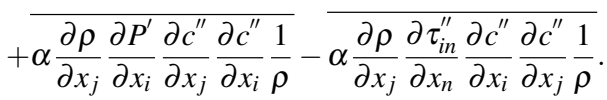

Replacing terms 1 and 2 in Eq. (14) leads to $\widetilde{\Delta}_{c}$ transport equation as given in Eq. (16).

\section{Appendix-B}

The scaling arguments explained in section 2 are applied to Eq. (14) to identify leading order terms. A number of simplifications have been made while carrying out the analysis. It is assumed that in the high $R e$ limit, the contributions from the viscous tensor will be small. Hence it is assumed that $\tau_{\text {in }} \approx 2 \mu S_{\text {in }}, \mu \approx \rho \alpha$ and the spatial gradients of density associated with $\tau_{\text {in }}$ are very small when compared with other terms.

The first term on the left-hand side of Eq. (16) can be decomposed into two parts as : 


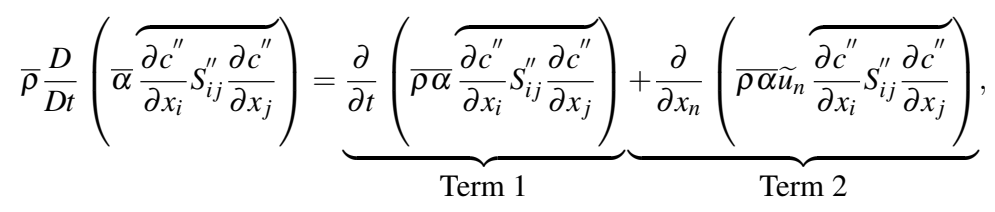

where

$$
\frac{\partial}{\partial t}\left(\overline{\rho \alpha} \widetilde{\frac{\partial c^{\prime \prime}}{\partial x_{i}} S_{i j}^{\prime \prime} \frac{\partial c^{\prime \prime}}{\partial x_{j}}}\right) \simeq \mathscr{O}\left(\rho_{u}\left(\frac{u_{L}^{0}}{\delta_{L}^{0}}\right)^{3} ; D a^{-1}\right)
$$

and

$$
\frac{\partial}{\partial x_{n}}\left(\overline{\rho \alpha} \widetilde{u}_{n} \widetilde{\frac{\partial c^{\prime \prime}}{\partial x_{i}} S_{i j}^{\prime \prime} \frac{\partial c^{\prime \prime}}{\partial x_{j}}}\right) \simeq \mathscr{O}\left(\rho_{u}\left(\frac{u_{L}^{0}}{\delta_{L}^{0}}\right)^{3} ;\left(\frac{u^{\prime} D a}{u_{r e f}}\right)^{-1}\right) .
$$

The first term on the right-hand side of Eq. (16) scales as:

$$
\frac{\partial}{\partial x_{n}}\left(\rho \alpha u_{n}^{\prime \prime} \frac{\partial c^{\prime \prime}}{\partial x_{i}} S_{i j}^{\prime \prime} \frac{\partial c^{\prime \prime}}{\partial x_{j}}\right) \simeq \mathscr{O}\left(\rho_{u}\left(\frac{u_{L}^{0}}{\delta_{L}^{0}}\right)^{3} ;\left(R e_{l_{t}} D a\right)^{-1 / 2}\right) .
$$

$D_{f 1}$ represents the diffusion/dissipation terms arising from the scalar part of the $\widetilde{\Delta}_{c}$ transport equation. Following Swaminathan and Bray [35] term $D_{f 1}$ can be expanded and simplified based on the assumption that the second gradient of $\rho$ is small compared with the other terms, thus leading to:

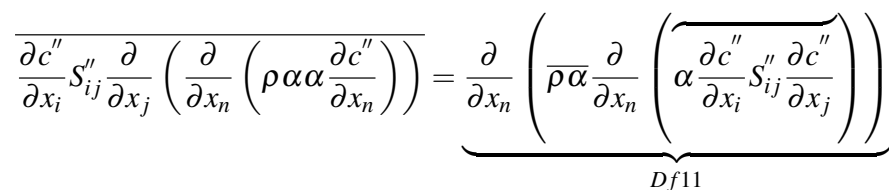

$$
\begin{aligned}
& \underbrace{-\rho \alpha \alpha \frac{\partial}{\partial x_{n}}\left(\frac{\partial c^{\prime \prime}}{\partial x_{j}}\right) \frac{\partial}{\partial x_{n}}\left(\frac{\partial c^{\prime \prime}}{\partial x_{i}} S_{i j}^{\prime \prime}\right)}_{D f 12} \underbrace{-\rho \alpha \alpha \frac{\partial}{\partial x_{n}\left(\frac{\partial c^{\prime \prime}}{\partial x_{i}} \frac{\partial c^{\prime \prime}}{\partial x_{j}} \frac{\partial}{\partial x_{n}} S_{i j}^{\prime \prime}\right)}}_{D f 13} \\
& \underbrace{-\rho \alpha \alpha \frac{\partial}{\partial x_{n}}\left(\frac{\partial c^{\prime \prime}}{\partial x_{j}} S_{i j}^{\prime \prime} \frac{\partial}{\partial x_{n}}\left(\frac{\partial c^{\prime \prime}}{\partial x_{i}}\right)\right)}_{D f 14} .
\end{aligned}
$$


Terms $D_{f 1 i}$ scale as :

$$
\begin{gathered}
D_{f 11} \simeq \mathscr{O}\left(\rho_{u}\left(\frac{u_{L}^{0}}{\delta_{L}^{0}}\right)^{3} ;\left(\operatorname{Dau}^{\prime}\right)^{-1}\right), \\
D_{f 12}, D_{f 13}, D_{f 14} \simeq \mathscr{O}\left(\rho_{u}\left(\frac{u_{L}^{0}}{\delta_{L}^{0}}\right)^{3} ; 1\right),
\end{gathered}
$$

Term $D_{f 2}$ represents the diffusion/dissipation from the momentum part of the $\widetilde{\Delta}_{c}$ transport equation and scales as:

$$
D_{f 2} \simeq \mathscr{O}\left(\rho_{u}\left(\frac{u_{L}^{0}}{\delta_{L}^{0}}\right)^{3} ; 1\right) .
$$

Terms $F_{1 i}$ representing the source terms scale as :

$$
F_{11}, F_{12} \simeq \mathscr{O}\left(\rho_{u}\left(\frac{u_{L}^{0}}{\delta_{L}^{0}}\right)^{3} ; 1\right)
$$

Terms $F_{2 i}$ representing the terms arising due to the turbulent transport of $\widetilde{\Delta}_{c}$ scale as :

$$
\begin{gathered}
F_{21}, F_{23} \simeq \mathscr{O}\left(\rho_{u}\left(\frac{u_{L}^{0}}{\delta_{L}^{0}}\right)^{3} ; \frac{u_{L}^{0} D a^{-1}}{u^{\prime}}\right), \\
F_{22} \simeq \mathscr{O}\left(\rho_{u}\left(\frac{u_{L}^{0}}{\delta_{L}^{0}}\right)^{3} ;\left(R e_{l_{t}} D a\right)^{-1}\right), \\
F_{24} \simeq \mathscr{O}\left(\rho_{u}\left(\frac{u_{L}^{0}}{\delta_{L}^{0}}\right)^{3} ;\left(\frac{u_{r e f} D a^{-3 / 2} R e_{l_{t}}^{-1 / 2}}{u^{\prime}}\right)\right) .
\end{gathered}
$$

Terms $F_{3 i}$ representing the dilatation terms scale as :

$$
\begin{aligned}
& F_{31}, F_{34}, F_{35}, F_{36} \simeq \mathscr{O}\left(\rho_{u}\left(\frac{u_{L}^{0}}{\delta_{L}^{0}}\right)^{3} ; 1\right), \\
& F_{32} \simeq \mathscr{O}\left(\rho_{u}\left(\frac{u_{L}^{0}}{\delta_{L}^{0}}\right)^{3} ;\left(\frac{u^{\prime} D a}{u_{r e f}}\right)^{-1}\right), \\
& F_{33} \simeq \mathscr{O}\left(\rho_{u}\left(\frac{u_{L}^{0}}{\delta_{L}^{0}}\right)^{3} ;\left(R e_{l_{t}} D a\right)^{-1 / 2}\right),
\end{aligned}
$$


Terms $F_{4 i}$ representing the turbulent straining terms scale as :

$$
\begin{gathered}
F_{41}, F_{44}, F_{45}, F_{48} \simeq \mathscr{O}\left(\rho_{u}\left(\frac{u_{L}^{0}}{\delta_{L}^{0}}\right)^{3} ; 1\right), \\
F_{42}, F_{46}, F_{47} \simeq \mathscr{O}\left(\rho_{u}\left(\frac{u_{L}^{0}}{\delta_{L}^{0}}\right)^{3} ;\left(\frac{u^{\prime} D a}{u_{r e f}}\right)^{-1}\right), \\
F_{43} \simeq \mathscr{O}\left(\rho_{u}\left(\frac{u_{L}^{0}}{\delta_{L}^{0}}\right)^{3} ;\left(\operatorname{Re}_{l_{t}} D a\right)^{-1 / 2}\right),
\end{gathered}
$$

\section{References}

1. Anderson, E., Bai, Z., Bischof, C., Blackford, S., Demmel, J., Dongarra, J., Du Croz, J., Greenbaum, A., Hammarling, S., McKenney, A., Sorensen, D.: LAPACK Users' Guide, third edn. Society for Industrial and Applied Mathematics, Philadelphia, PA (1999)

2. Ashurst, W.T., Kerstein, a.R., Kerr, R.M., Gibson, C.H.: Alignment of vorticity and scalar gradient with strain rate in simulated Navier Stokes turbulence. Physics of Fluids 30(8), 2343 (1987). DOI $10.1063 / 1.866513$

3. Batchelor, G.: The effect of homogeneous turbulence on material lines and surfaces. Proceedings of the Royal Society of London. Series A, Mathematical and Physical Sciences 213(1114), 349-366 (1952)

4. Borghi, R.: Turbulent premixed combustion: Further discussions on the scales of fluctuations. Combustion and Flame 80(3-4), 304-312 (1990). DOI 10.1016/0010-2180(90)90106-2

5. Bray, K.: Modelling methods, Laminar flamelets and the Bray Moss Libby model. In: N. Swaminathan, K.N.C. Bray (eds.) Turbulent Premixed Flames, pp. 42-60. Cambridge University Press (2011)

6. Bray, K.N.C.: The interaction between turbulence and combustion. Symposium (International) on Combustion 17(1), 223-233 (1979). DOI 10.1016/S0082-0784(79)80024-7

7. Bray, K.N.C.: Studies of the Turbulent Burning Velocity. Proceedings: Mathematical and Physical Sciences 431(1882), 315-335 (1990)

8. Bray, K.N.C., Libby, P.A., Moss, J.B.: Unified modeling approach for premixed turbulent combustionPart I: General formulation. Combustion and Flame 61(1), 87-102 (1985). DOI 10.1016/00102180(85)90075-6 
9. Bray, K.N.C., Moss, J.B.: A Unified Statistical Model Of The Premixed Turbulent Flame. Acta Astronautica 4(3-4), 291-319 (1977). DOI 10.1016/0094-5765(77)90053-4

10. Candel, S., Poinsot, T.: Flame Stretch and the Balance Equation for the Flame Area. Combustion Science and Technology 70(1), 1-15 (1990). DOI 10.1080/00102209008951608

11. Cant, R.S.: SENGA2 User Guide, CUED/A THERMO/TR67. Tech. rep., University Of Cambridge, Cambridge, United Kingdom (2012)

12. Cant, R.S., Mastorakos, E.: An introduction to turbulent reacting flows. Imperial College Press (2008)

13. Chakraborty, N., Champion, M., Mura, A., Swaminathan, N.: Modelling methods, Scalar dissipation rate approach. In: N. Swaminathan, K.N.C. Bray (eds.) Turbulent Premixed Flames, pp. 74-102. Cambridge University Press (2011)

14. Chakraborty, N., Rogerson, J.W., Swaminathan, N.: A priori assessment of closures for scalar dissipation rate transport in turbulent premixed flames using direct numerical simulation. Physics of Fluids 20(4), 045,106 (2008). DOI 10.1063/1.2903846

15. Chakraborty, N., Swaminathan, N.: Influence of the Damkohler number on turbulence-scalar interaction in premixed flames. I. Physical insight. Physics of Fluids 19(4), 045,103 (2007). DOI 10.1063/1.2714070

16. Chakraborty, N., Swaminathan, N.: Influence of the Damkohler number on turbulence-scalar interaction in premixed flames. II. Model development. Physics of Fluids 19(4), 045,104 (2007). DOI $10.1063 / 1.2714076$

17. Chakraborty, N., Swaminathan, N.: Reynolds Number Effects on Scalar Dissipation Rate Transport and Its Modeling in Turbulent Premixed Combustion. Combustion Science and Technology 185(4), 676-709 (2013). DOI 10.1080/00102202.2012.741635

18. Dunstan, T.D.: Turbulent premixed flame kernel growth during the early stages using Direct Numerical Simulation. Ph.D. thesis, Cranfield University (2008)

19. Dunstan, T.D., Minamoto, Y., Chakraborty, N., Swaminathan, N.: Scalar dissipation rate modelling for Large Eddy Simulation of turbulent premixed flames. Proceedings of the Combustion Institute 34(1), 1193-1201 (2013). DOI 10.1016/j.proci.2012.06.143

20. Dunstan, T.D., Swaminathan, N., Bray, K.N.C.: Influence of flame geometry on turbulent premixed flame propagation: a DNS investigation. Journal of Fluid Mechanics 709, 191-222 (2012). DOI $10.1017 / \mathrm{jfm} .2012 .328$ 
21. Dunstan, T.D., Swaminathan, N., Bray, K.N.C., Cant, R.S.: Geometrical properties and turbulent flame speed measurements in stationary premixed V-flames using Direct Numerical Simulation. Flow, Turbulence and Combustion 87(2-3), 237-259 (2010). DOI 10.1007/s10494-010-9284-1

22. Hartung, G., Hult, J., Kaminski, C., Rogerson, J.W., Swaminathan, N.: Effect of heat release on turbulence and scalar-turbulence interaction in premixed combustion. Physics of Fluids 20(3), 035,110 (2008). DOI $10.1063 / 1.2896285$

23. Jenkins, K., Cant, R.: Direct numerical simulation of turbulent flame kernels. In: D. Knight, L. Sakell (eds.) Recent Advances in DNS and LES: Proceedings of the Second AFOSR Conference, Rutgers - The State University of New Jersey, New Brunswick, USA, pp. 191-202. Kluwer, Dordrecht (1999)

24. Kim, S.H., Pitsch, H.: Scalar gradient and small-scale structure in turbulent premixed combustion. Physics of Fluids 19(11), 115,104 (2007). DOI 10.1063/1.2784943

25. Kolla, H., Rogerson, J.W., Chakraborty, N., Swaminathan, N.: Scalar dissipation rate modeling and its validation. Combustion Science and Technology 181(3), 518-535 (2009). DOI $10.1080 / 00102200802612419$

26. Liu, Y., Dowling, A.P., Swaminathan, N., Dunstan, T.D.: Spatial correlation of heat release rate and sound emission from turbulent premixed flames. Combustion and Flame 159(7), 2430-2440 (2012). DOI 10.1016/j.combustflame.2012.03.003

27. Minamoto, Y., Fukushima, N., Tanahashi, M., Miyauchi, T., Dunstan, T.D., Swaminathan, N.: Effect of flow-geometry on turbulence-scalar interaction in premixed flames. Physics of Fluids 23(12), 125,107 (2011). DOI 10.1063/1.3665619

28. Poinsot, T., Veynante, D.: Theoretical and Numerical Combustion, 2nd edn. R.T.Edwards, Inc (2005)

29. Poinsot, T.J., Candel, S., Trouvé, A.: Applications of direct numerical simulation to premixed turbulent combustion. Progress in Energy and Combustion Science 21(6), 531-576 (1995). DOI 10.1016/03601285(95)00011-9

30. Pope, S.B.: The evolution of surfaces in turbulence. International Journal of Engineering Science 26(5), 445-469 (1988). DOI 10.1016/0020-7225(88)90004-3

31. Prosser, R.: Improved boundary conditions for the direct numerical simulation of turbulent subsonic flows. I. Inviscid flows. Journal of Computational Physics 207(2), 736-768 (2005). DOI 10.1016/j.jcp.2005.01.027

32. Prosser, R.: Improved boundary conditions for the DNS of reacting subsonic flows. Flow, Turbulence and Combustion 87(2-3), 351-376 (2010). DOI 10.1007/s10494-010-9307-y 
33. Steinberg, A.M., Driscoll, J.F., Swaminathan, N.: Statistics and dynamics of turbulence-flame alignment in premixed combustion. Combustion and Flame 159(8), 2576-2588 (2012). DOI 10.1016/j.combustflame.2011.12.001

34. Sutherland, J.C., Kennedy, C.a.: Improved boundary conditions for viscous, reacting, compressible flows. Journal of Computational Physics 191(2), 502-524 (2003). DOI 10.1016/S0021-9991(03)00328-0

35. Swaminathan, N., Bray, K.N.C.: Effect of dilatation on scalar dissipation in turbulent premixed flames. Combustion and Flame 143(4), 549-565 (2005). DOI 10.1016/j.combustflame.2005.08.020

36. Swaminathan, N., Grout, R.W.: Interaction of turbulence and scalar fields in premixed flames. Physics of Fluids 18(4), 045,102 (2006). DOI 10.1063/1.2186590

37. Yoo, C.S., Im, H.G.: Characteristic boundary conditions for simulations of compressible reacting flows with multi-dimensional, viscous and reaction effects. Combustion Theory and Modelling 11(2), 259-286 (2007). DOI 10.1080/13647830600898995 


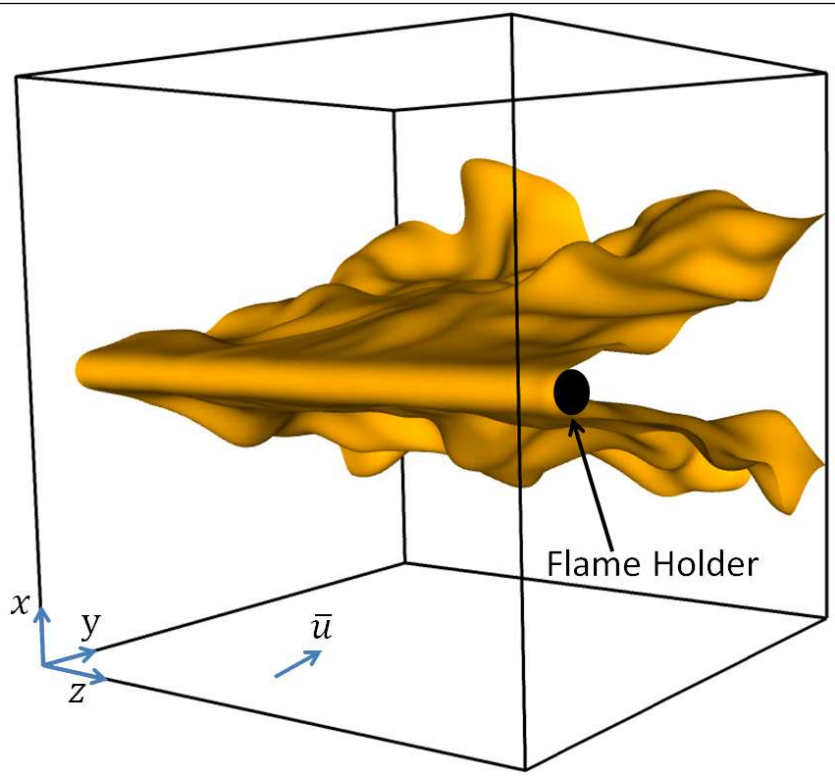

Fig. 1 Computational domain for the V-flame DNS

\begin{tabular}{|c|c|c|c|c|c|}
\hline$u_{\text {in }}^{\prime} / u_{L}^{0}$ & $\bar{u}_{\text {in }} / u_{L}^{0}$ & $R e_{l_{t}, \text { in }}$ & $l_{t, \text { in }} / \delta_{L}^{0}$ & $K a_{\text {in }}$ & $D a_{\text {in }}$ \\
\hline 2.0 & 16.6 & 37 & 12.82 & 0.79 & 6.41 \\
\hline
\end{tabular}

Table 1 DNS database parameters at inlet plane

\begin{tabular}{|c|c|c|c|c|c|}
\hline Sampling location & $u^{\prime} / u_{L}^{0}$ & $R e_{l_{t}}$ & $l_{t} / \delta_{L}^{0}$ & $K a$ & $D a$ \\
\hline Region a & 1.51 & 22.16 & 10.0 & 0.50 & 9.94 \\
\hline Region b & 1.48 & 17.56 & 8.6 & 0.49 & 9.09 \\
\hline Region c & 1.41 & 22.16 & 9.8 & 0.46 & 10.31 \\
\hline
\end{tabular}

Table 2 DNS database parameters at the sampling locations

\begin{tabular}{|c|c|c|c|c|}
\hline Sampling location & $R e_{l_{t}} \times D a$ & $n_{e}$ & $n_{g}$ & $2(1+\tau)^{0.7}\left(\frac{N x}{n_{g}} n_{e}\right)^{2}$ \\
\hline Region a & 220.3 & 1.48 & 28 & 889 \\
\hline Region b & 159.62 & 1.72 & 30 & 1047 \\
\hline Region c & 228.47 & 1.51 & 29 & 863 \\
\hline
\end{tabular}

Table 3 The numerical resolution criterion of Poinsot et al [29] 


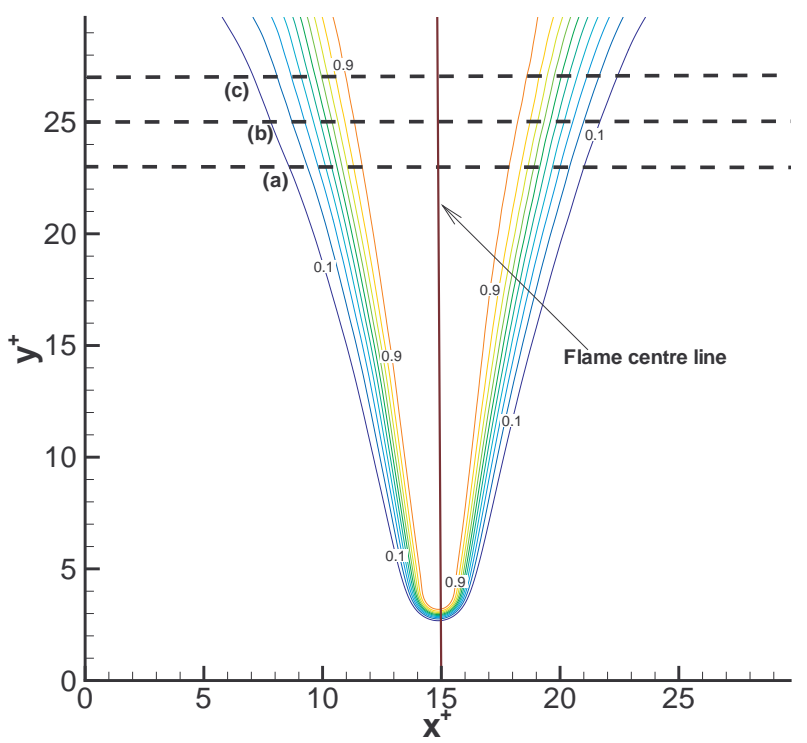

Fig. 2 Favre averaged progress variable $\widetilde{c}$ contours $0.1-0.9$. The dashed lines represent the sampling locations, and the solid line represents the flame centre line. 


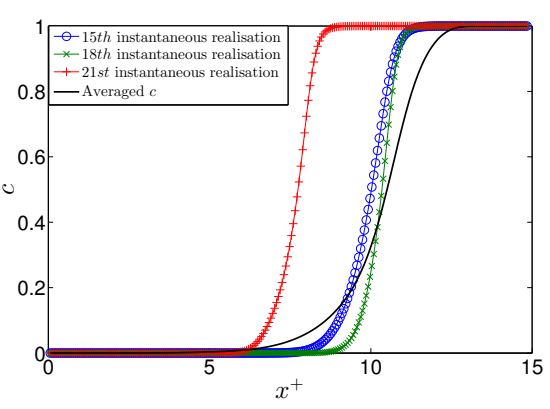

(a) $y^{+} \approx 23$

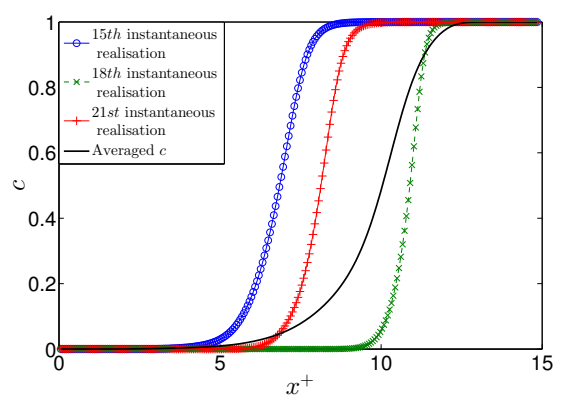

(b) $y^{+} \approx 25$

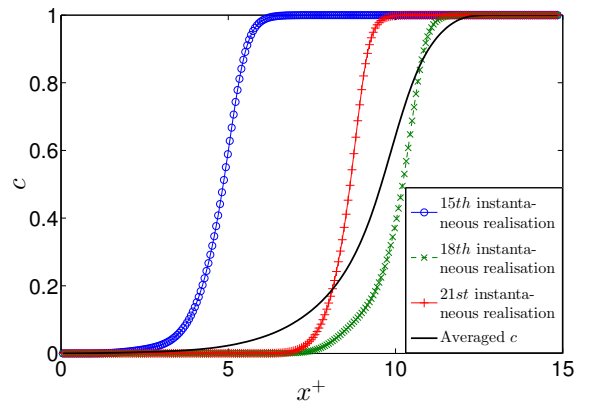

(c) $y^{+} \approx 27$

Fig. 3 Spatial variation of instantaneous progress variable at different times compared with the Favre averaged progress variable. The symbols represent exact grid points inside the domain. 


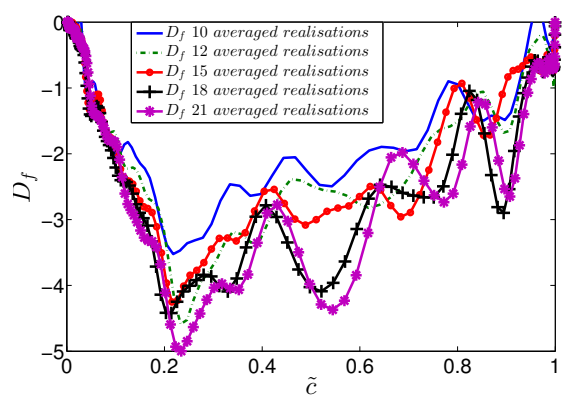

(a) $y^{+} \approx 23$

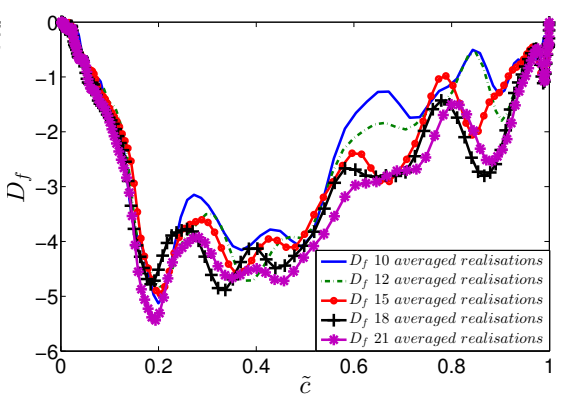

(b) $y^{+} \approx 25$

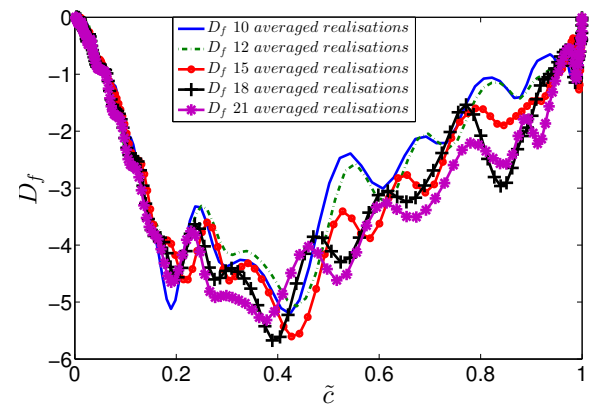

(c) $y^{+} \approx 27$

Fig. 4 Convergence of $D_{f}$ in time for different realisations of the flow field. The values are normalised using the respective $\rho_{u}, u_{L}^{0}$ and $\delta_{L}^{0}$. 


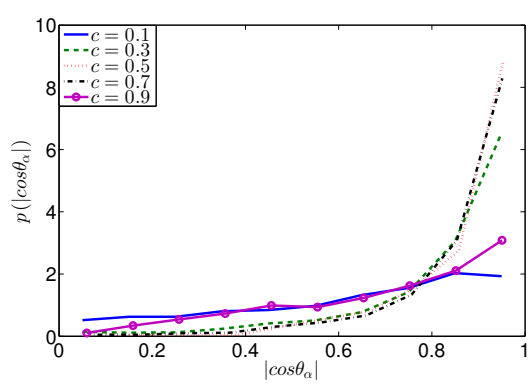

(a) Direction cosine between $e_{\alpha}$ and $\nabla c$

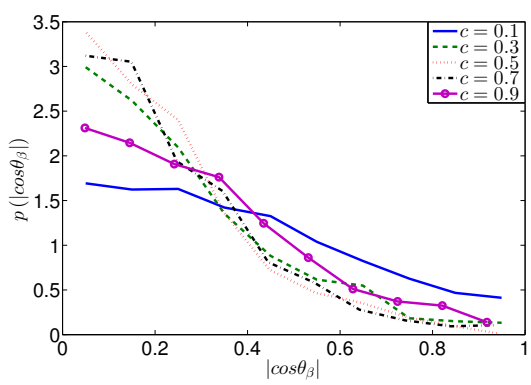

(c) Direction cosine between $e_{\beta}$ and $\nabla c$

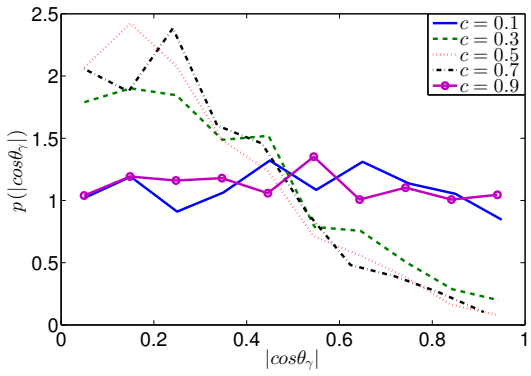

(e) Direction cosine between $e_{\gamma}$ and $\nabla c$

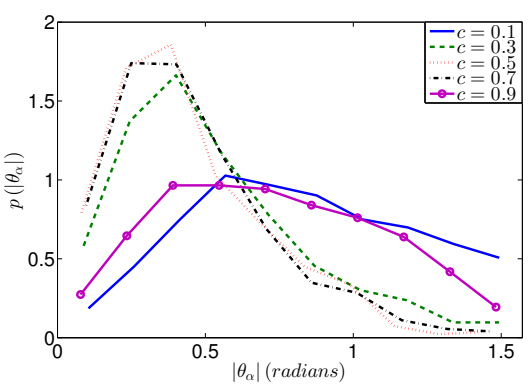

(b) The angle between $e_{\alpha}$ and $\nabla c$

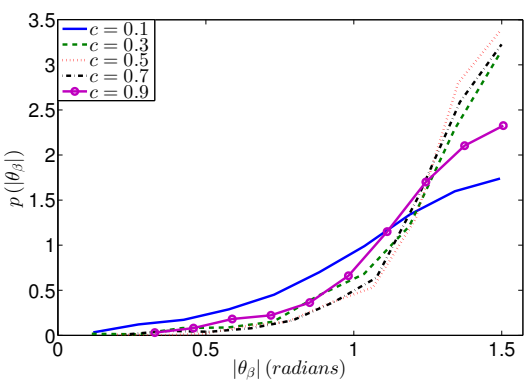

(d) The angle between $e_{\beta}$ and $\nabla c$

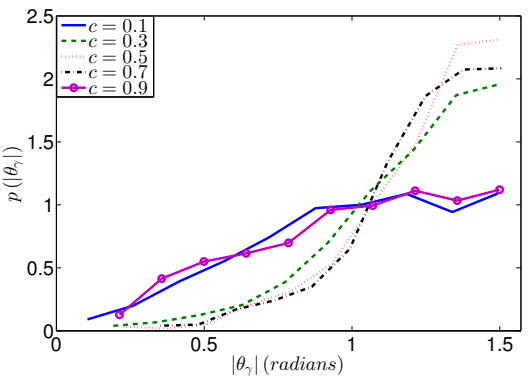

(f) The angle between $e_{\gamma}$ and $\nabla c$

Fig. 5 Pdfs of the direction cosines and the associated angles at $y^{+} \approx 23$ 

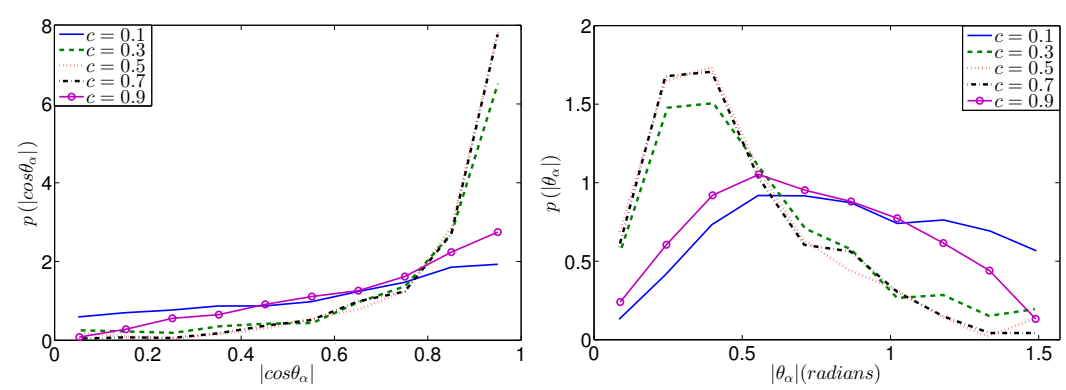

(a) Direction cosine between $e_{\alpha}$ and $\nabla c$

(b) The angle between $e_{\alpha}$ and $\nabla c$
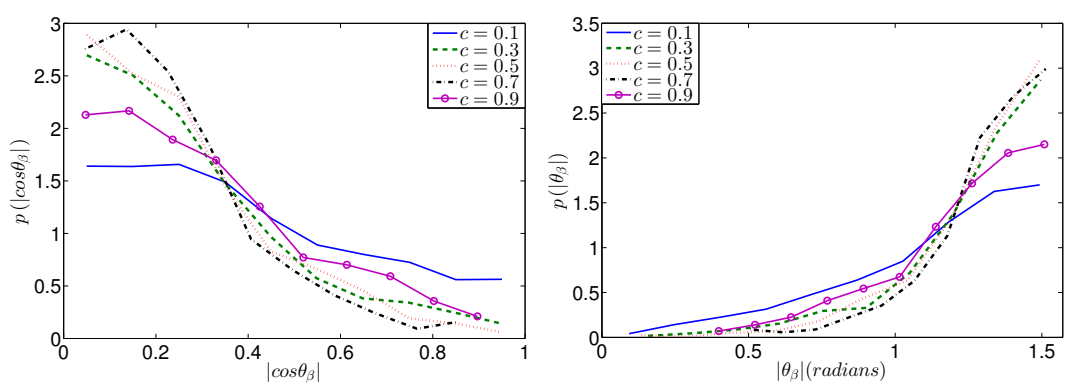

(c) Direction cosine between $e_{\beta}$ and $\nabla c$

(d) The angle between $e_{\beta}$ and $\nabla c$
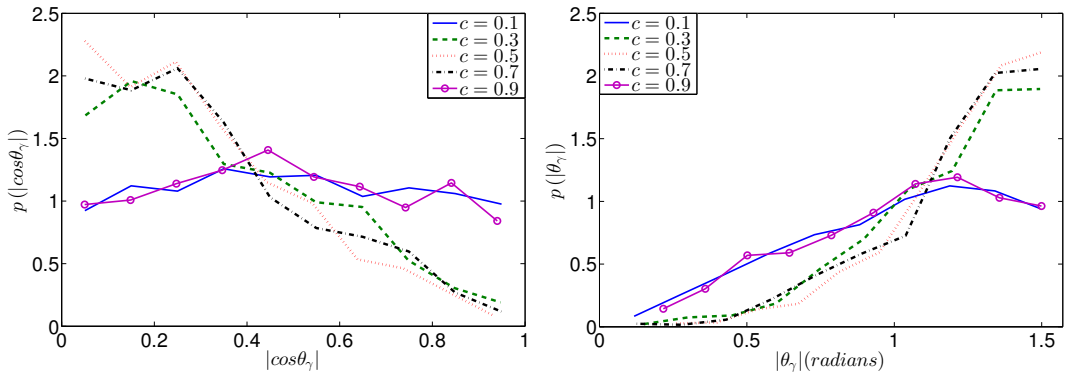

(e) Direction cosine between $e_{\gamma}$ and $\nabla c$

(f) The angle between $e_{\gamma}$ and $\nabla c$

Fig. 6 Pdfs of the direction cosines and the associated angles at $y^{+} \approx 25$ 

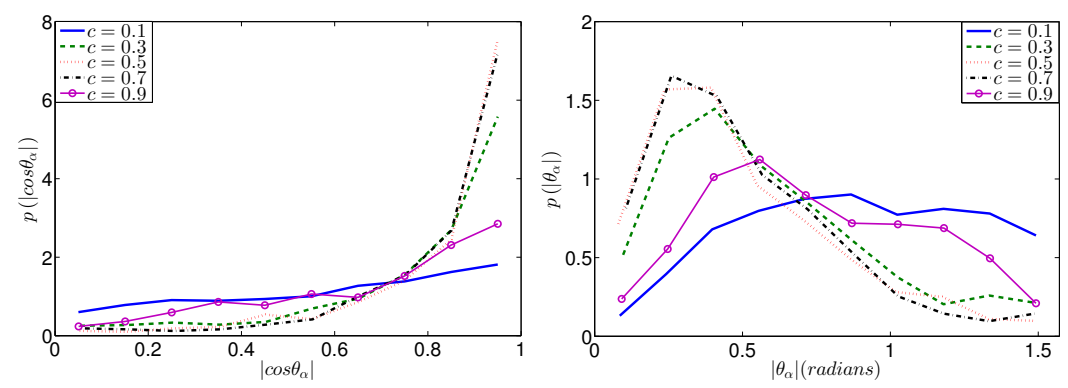

(a) Direction cosine between $e_{\alpha}$ and $\nabla c$

(b) The angle between $e_{\alpha}$ and $\nabla c$
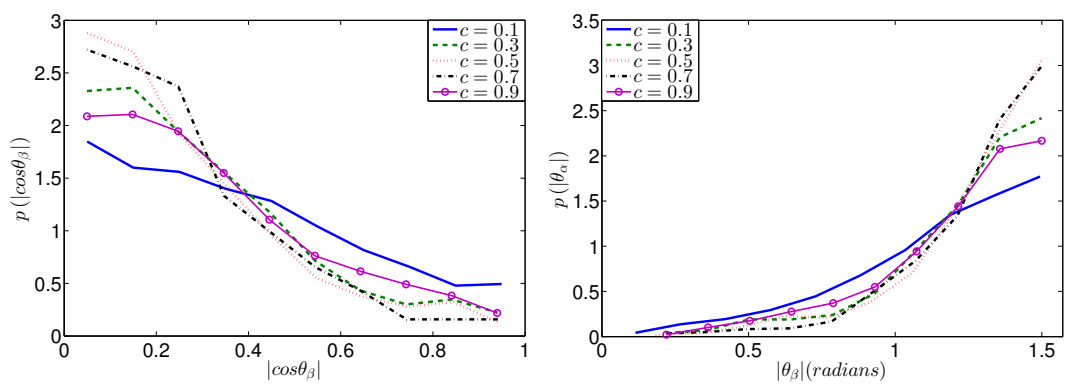

(c) Direction cosine between $e_{\beta}$ and $\nabla c$

(d) The angle between $e_{\beta}$ and $\nabla c$
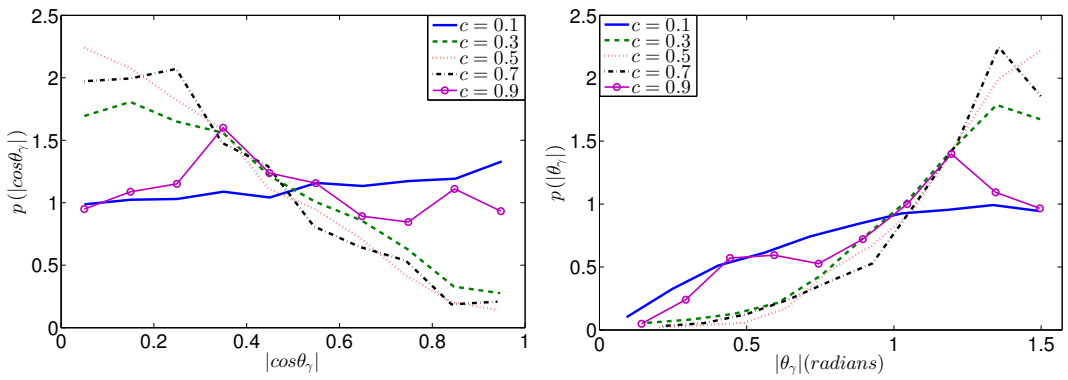

(e) Direction cosine between $e_{\gamma}$ and $\nabla c$

(f) The angle between $e_{\gamma}$ and $\nabla c$

Fig. 7 Pdfs of the direction cosines and the associated angles at $y^{+} \approx 27$ 


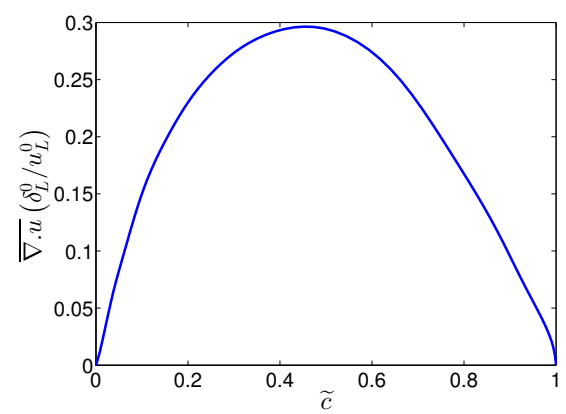

(a) $y^{+} \approx 23$

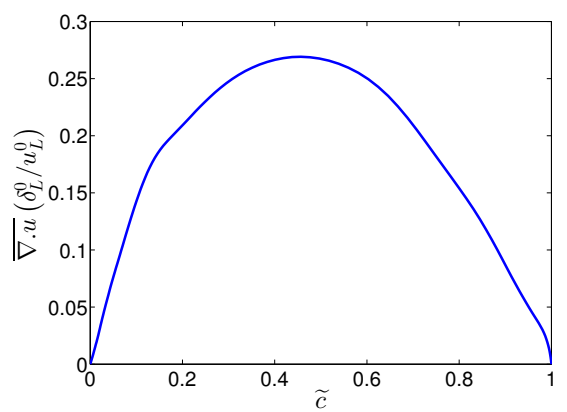

(b) $y^{+} \approx 25$

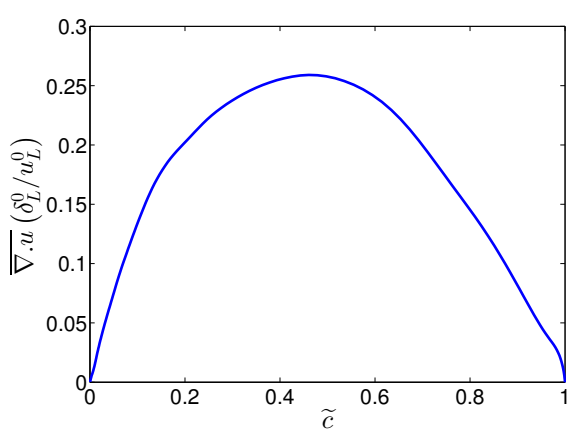

(c) $y^{+} \approx 27$

Fig. 8 Normalised dilatation in the V-flame DNS 

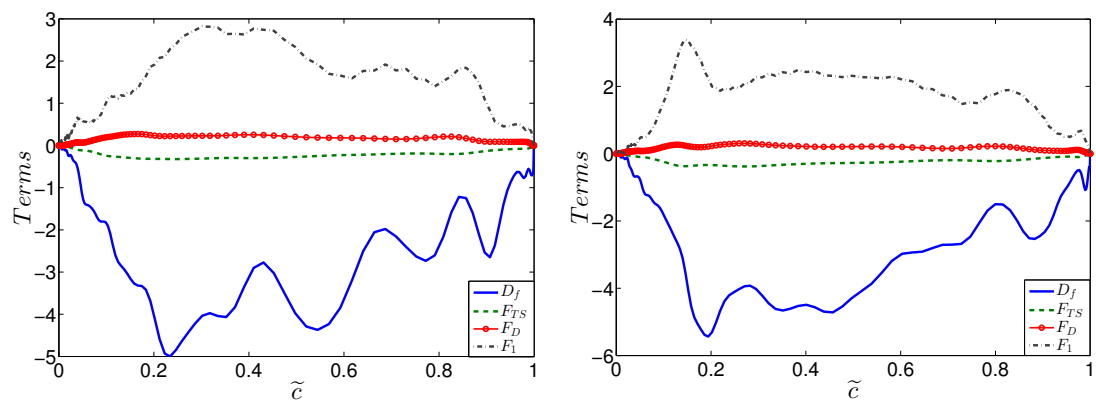

(a) $y^{+} \approx 23$

(b) $y^{+} \approx 25$

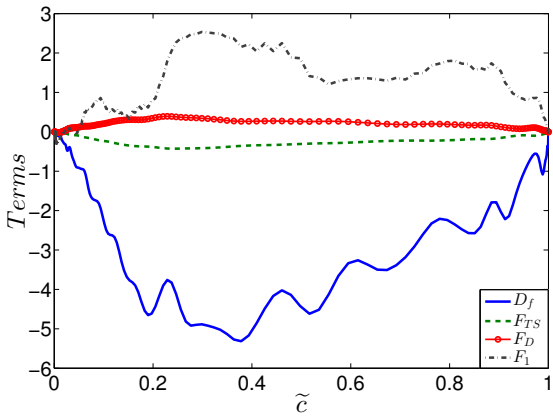

(c) $y^{+} \approx 27$

Fig. 9 Leading order terms. The values are normalised using the respective $\rho_{u}, u_{L}^{0}$ and $\delta_{L}^{0}$. 


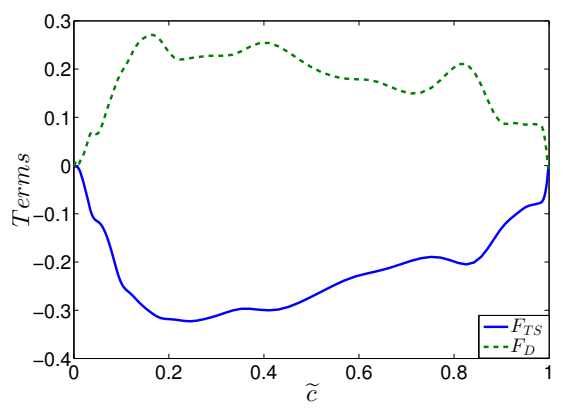

(a) $y^{+} \approx 23$

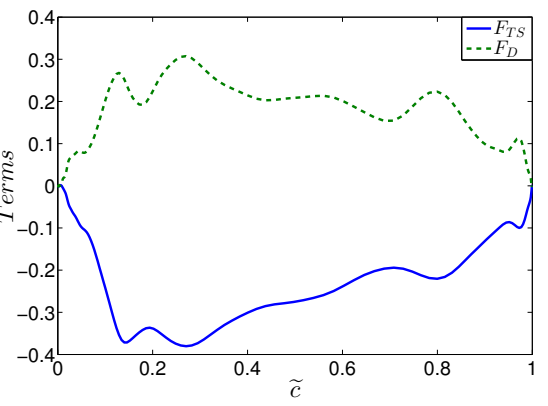

(b) $y^{+} \approx 25$

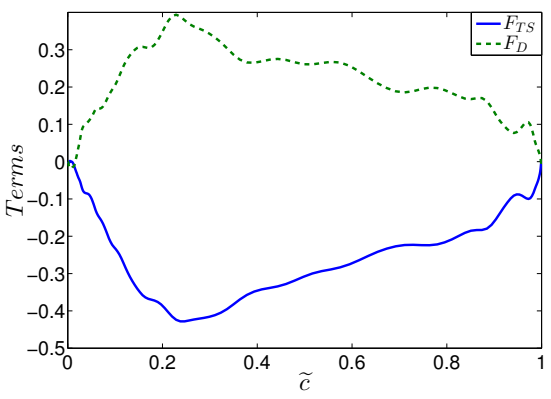

(c) $y^{+} \approx 27$

Fig. 10 Leading order terms without the diffusion/dissipation and source terms. The values are normalised using the respective $\rho_{u}, u_{L}^{0}$ and $\delta_{L}^{0}$. 


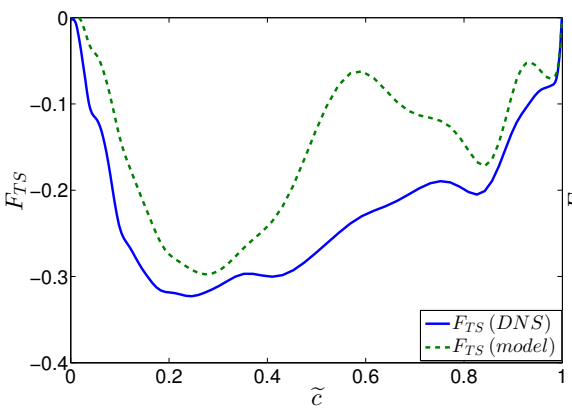

(a) $y^{+} \approx 23$

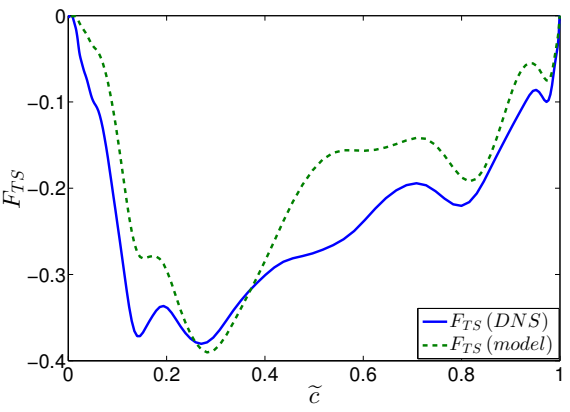

(b) $y^{+} \approx 25$

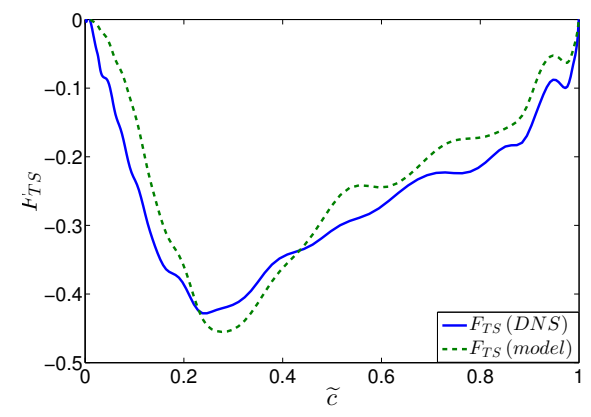

(c) $y^{+} \approx 27$

Fig. 11 Comparisons of $F_{T S}$ model predictions and the DNS results. The values are normalised using the respective $\rho_{u}, u_{L}^{0}$ and $\delta_{L}^{0}$. 


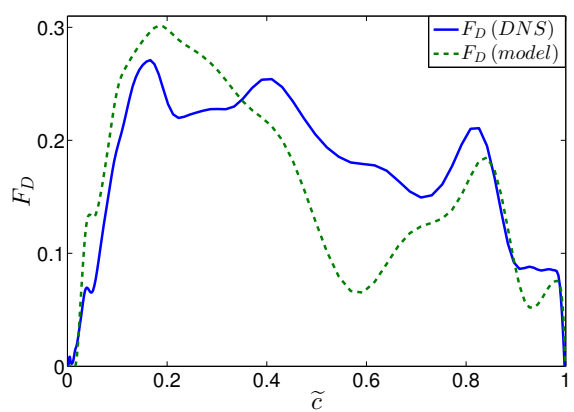

(a) $y^{+} \approx 23$

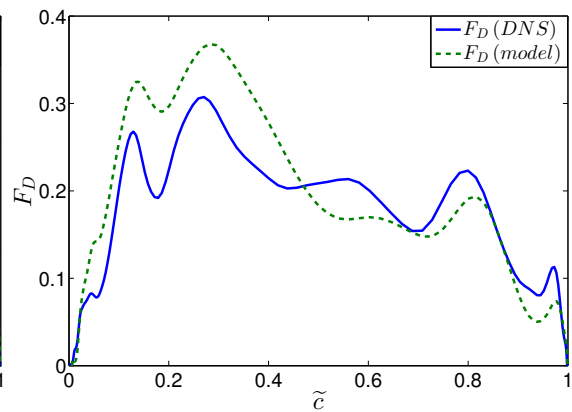

(b) $y^{+} \approx 25$

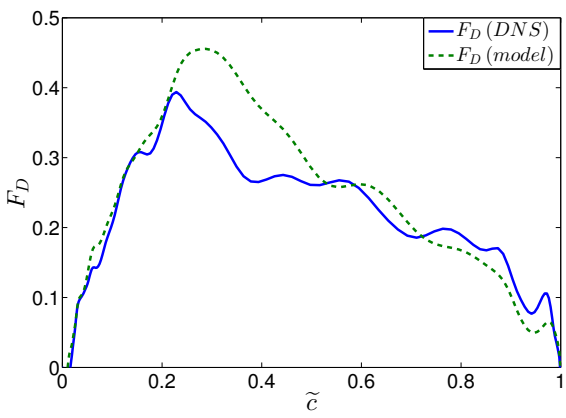

(c) $y^{+} \approx 27$

Fig. 12 Comparisons of $F_{D}$ model predictions and the DNS results. The values are normalised using the respective $\rho_{u}, u_{L}^{0}$ and $\delta_{L}^{0}$. 


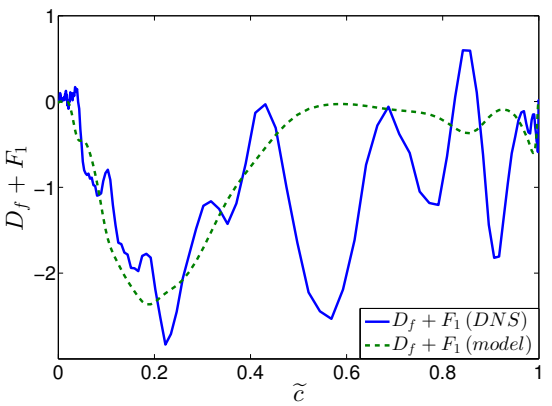

(a) $y^{+} \approx 23$

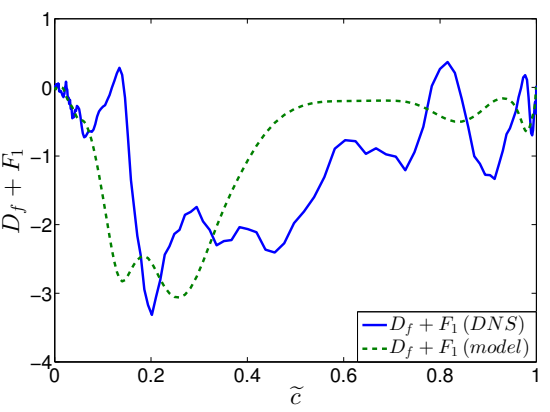

(b) $y^{+} \approx 25$

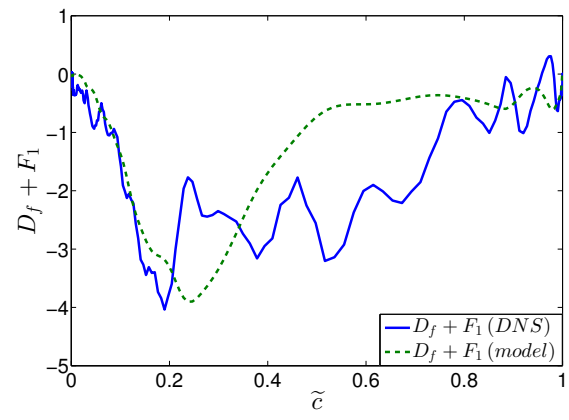

(c) $y^{+} \approx 27$

Fig. 13 Comparisons of $\left(D_{f}+F_{1}\right)$ model predictions and the DNS results. The values are normalised using the respective $\rho_{u}, u_{L}^{0}$ and $\delta_{L}^{0}$. 